\title{
Effect of temperature and hydrophilic ratio on the structure of poly(N-vinylcaprolactam)-block-poly(dimethylsiloxane)-block- poly(N-vinylcaprolactam) polymersomes
}

\author{
Yiming Yang ${ }^{1, \&}$, Aaron Alford ${ }^{1, \&}$, Veronika Kozlovskaya ${ }^{1}$, Shidi Zhao ${ }^{2}$, Himanshu Joshi ${ }^{2}$, \\ Eunjung Kim ${ }^{3}$, Shuo Qian ${ }^{4}$, Volker Urban ${ }^{4}$, Donald Cropek ${ }^{3}$, Aleksei Aksimentiev², Eugenia \\ Kharlampieva ${ }^{1,5},{ }^{*}$ \\ ${ }^{1}$ Department of Chemistry, University of Alabama at Birmingham, Birmingham, Alabama 35205, \\ United States \\ ${ }^{2}$ Department of Physics, Beckman Institute, University of Illinois at Urbana Champaign, Urbana, \\ Illinois 61801, United States \\ ${ }^{3}$ U.S. Army Engineer Research and Development Center, Construction Engineering Research \\ Laboratory, Champaign, Illinois 61822, United States \\ ${ }^{4}$ Neutron Scattering Division, Neutron Sciences Directorate, Oak Ridge National Laboratory, Oak \\ Ridge, Tennessee 37831, United States \\ ${ }^{5}$ Center for Nanoscale Materials and Biointegration, University of Alabama at Birmingham, \\ Birmingham, Alabama 35205, United States
}

\begin{abstract}
Nanosized polymeric vesicles (polymersomes) assembled from ABA triblock copolymers of poly(N-vinylcaprolactam)-poly(dimethylsiloxane)-poly(N-vinylcaprolactam) (PVCL-PDMSPVCL) are a promising platform for biomedical applications, as the temperature-responsiveness of the PVCL blocks enables reversible vesicle shrinkage and permeability of the polymersome shell at elevated temperatures. Herein, we explore the effects of molecular weight, polymer block weight ratios, and temperature on the structure of these polymersomes via electron microscopy, dynamic light scattering, small angle neutron scattering (SANS), and all-atom molecular dynamic methods. We show that the shell structure and overall size of the polymersome can be tuned by varying the hydrophilic (PVCL) weight fraction of the polymer: at room temperature, polymers of
\end{abstract}

\footnotetext{
*Corresponding Author ekharlam@uab.edu.

$\&$ Author Contributions

Y.Y. and A.A. contributed equally to this work

ASSOCIATED CONTENT

Supporting Information.

The following file is available free of charge. GPC data for PVCL-PDMS-PVCL and PMOXA-PDMS-PMOXA, GPC traces for PVCL-PDMS-PVCL copolymers, and cloud point determinations of PVCL homopolymer solutions; ${ }^{1} \mathrm{H}$ NMR spectra for bis(hydroxyethyl)-oxypropyl poly(dimethylsiloxane) (HO-PDMS $30-\mathrm{OH}$ ), the product of end group decoration (Br-PDMS $30-\mathrm{Br}$ ), and the final macro initiator (X-PDMS $30-\mathrm{X}$ ); fit parameters obtained from the poly core-shell model deduced from SANS curves; cryoTEM of PVCL-PDMS-PVCL copolymers; End-to-end distance of PVCL5, PVCL7; PVCL10 single block systems, snapshots of MD trajectories of PVCL 5 and PVCL 10 at $275 \mathrm{~K}$ and $328 \mathrm{~K}$, and simulation videos illustrating the collapsing behavior of PVCL5 at $275 \mathrm{~K}$ and $328 \mathrm{~K}$ (movies 1-2).

The authors declare no competing financial interest.
} 
smaller hydrophilic ratios form larger vesicles that have thinner shells, whereas polymers with higher PVCL content exhibit interchain aggregation of PVCL blocks within the polymersome shell above $50{ }^{\circ} \mathrm{C}$. Model fitting and model-free analysis of the SANS data reveals that increasing the mass ratio of PVCL to the total copolymer weight from 0.3 to 0.56 reduces the temperatureinduced change in vesicle diameter by a factor of 3 while simultaneously increasing the change in shell thickness by a factor of 1.5. Finally, by analysis of the shell structures and overall size of polymersomes with various PVCL weight ratios and those without temperature-dependent polymer components, we bring into focus the mechanism of temperature-triggered drug release reported in a previous study. This work provides new fundamental perspectives on temperatureresponsive polymersomes and elucidates important structure-property relationships of their constituent polymers.

\section{Graphical Abstract}

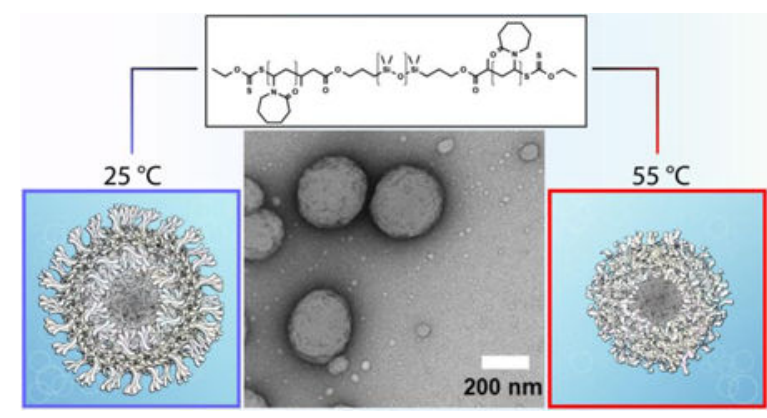

\section{Keywords}

Polymersomes; Temperature Responsive; SANS; Molecular Dynamics; Poly(N-vinylcaprolactam); Triblock copolymer

\section{Introduction}

Naturally occurring self-assembled biological carriers (e.g., cell membranes, exosomes, and mitochondria) that provide a nanometer-thin, bubble-like hydrophobic barrier to protect and transport cargo have inspired the synthesis and study of new polymeric vesicles with customizable properties for advancing the field of precision therapy. ${ }^{1-6}$ Because of the high degree of control over their structure and function, the design of self-assembled polymeric vesicles (polymersomes) is rapidly advancing as they can help mitigate the side effects of systemic drug delivery and significantly improve drug efficacy by providing precise control over the space and time within which drugs are released. ${ }^{711}{ }^{\mathbb{P}}$

Despite the morphology flexibility of the liposomal vesicles has been shown to facilitate liposomal skin penetrating ability, the liposomes can be destroyed under flow conditions such as those found in the bloodstream due to their low mechanical stability. ${ }^{12-14}$ Conversely, regardless of the assembly method, polymersomes demonstrate increased mechanical stability compared to liposomes, efficient drug entrapment, long shelf life, and controllable stimuli-triggered delivery of cargo.${ }^{15-21}$ In addition, although temperature can be used as a trigger for drug release from nanovesicles due to the elevated temperature in 
tumor microenvironments because of the high rate of tumor metabolism, ${ }^{22}$ imbuing liposomes with precise temperature sensitivity in biologically relevant ranges can be difficult due to the complex nature of their formulations, ${ }^{23,24}$ as well as controlling their size to promote predictable ensemble behavior can be challenging due to liposome propensity for wide size distributions upon self-assembly. ${ }^{25}$

Therefore, the development of polymersomes that are mechanically robust, have efficient drug loading capacity, and can intelligently respond to environmental stimuli such as temperature or $\mathrm{pH}$, has become of great importance. ${ }^{26-30}$ Thus, $\mathrm{pH}$-responsive polymer vesicles made from block copolymers of polybutadiene- $b$-poly(L-glutamic acid) and polyethyloxide- $b$-polycaprolactam have been shown to release their cargo through the vesicle disassembly because of degradable bonds within block copolymer structure. ${ }^{27,28}$ In contrast, temperature as a stimulus is much easier to control via external means and can be used to precisely control the rate of cargo release if polymer blocks with lower critical solution temperature (LCST) are included in the polymersome architecture. ${ }^{31}$ Poly $(\mathrm{N}$ isopropylacrylamide) (PNIPAM) is a well-known polymer with a discontinuous LCST transition which is often used as a component of temperature responsive structures. ${ }^{32,33}$ For example, McCormick et al have shown a temperature-responsive polymersome from $\mathrm{P}\left(\right.$ DMAEMA $_{165}$-b-NIPAM ${ }_{435}$ ) with incorporated gold nanoparticles that could disassemble on demand at high temperatures. ${ }^{34}$ Yang et al have demonstrated that poly(ethylene oxide)block-poly( $\mathrm{N}$-isopropylacrylamide) copolymers assemble into vesicles in solution above $37^{\circ} \mathrm{C}$ and slowly released their cargo upon cooling to room temperature and subsequent disassembly..$^{33}$

However, the vesicle self-assembly at $\mathrm{T}>36^{\circ} \mathrm{C}$ have limited use for biomedical therapy as they require a temperature decrease, often lower than physiologically relevant temperatures, to disassemble the polymer vesicle membrane and release the therapeutic cargo. ${ }^{32}$ In addition, to retain structural integrity, vesicles assembled above the polymer LCST are often chemically crosslinked, which limits their biodegradability. ${ }^{27}$

We have recently shown that poly(N-vinylcaprolactam)-poly(dimethylsiloxane)-poly(Nvinylcaprolactam) $\left(\mathrm{PVCL}_{\mathrm{n}}-\mathrm{PDMS}_{65}-\mathrm{PVCL}_{\mathrm{n}}\right)$ copolymers with $\mathrm{n}=10,15,19$ can assemble into stable polymersomes at room temperature when the ratio $f$ of the hydrophilic PVCL block to the total polymer mass is $0.36 \leq f \leq 0.52 .{ }^{31}$ Importantly, owing to the temperaturetriggered spinodal decomposition (LCST behavior) of PVCL, the PVCL chains in our copolymer exhibit hydrophilic characteristics at room temperature but undergo a coilglobule transition above the phase transition temperature. ${ }^{28}$ Based on this behavior, we previously showed that increasing the temperature above a critical point results in a reversible shrinkage of the polymersomes without compromising the structural stability of the vesicles. ${ }^{31}$ To demonstrate their potential for drug delivery applications, we reported in the above study that these polymersomes showed temperature-dependent permeability toward the model drug doxorubicin (DOX; MW = 544 Da) which was encapsulated in the hydrophilic cavity of the polymersome. However, despite the potential of these $\mathrm{PVCL}_{\mathrm{n}}{ }^{-}$ $\mathrm{PDMS}_{\mathrm{m}}-\mathrm{PVCL}_{\mathrm{n}}$ polymersomes as drug carriers due to their structural stability and degradability at lower $\mathrm{pH}$ values, ${ }^{31}$ little is known about the structural changes of the 
$\mathrm{PVCL}_{\mathrm{n}}-\mathrm{PDMS}_{\mathrm{m}}-\mathrm{PVCL}_{\mathrm{n}}$ polymersome membrane over the temperature responsive range (25 $-45^{\circ} \mathrm{C}$ ) which lead to temperature-induced vesicle size and permeability changes.

To characterize self-assembled systems, dynamic/static light scattering ${ }^{29}$ can be used to evaluate vesicle size distribution, critical aggregation concentration, and the associated response to temperature or $\mathrm{pH}$ change, ${ }^{36,37,38}$ while optical microscopy may only resolve giant vesicles with a diameter larger than $1 \mu \mathrm{m} .{ }^{38,39,40}$ In situ small-angle neutron scattering (SANS) is the most relevant technique for characterizing micron and nanometer scale polymersomes as it allows for studying polymersomes in solutions at a wide range of temperatures with angstrom resolution. ${ }^{41,42}$ The neutron beam is non-destructive and reveals contrast between isotopes of hydrogen, allowing structural characterization of the hydrogenrich polymersome membrane if assembled in $\mathrm{D}_{2} \mathrm{O} .{ }^{43}$ Recently, in situ SANS elucidated the temperature-responsive behavior of poly(ethyl glycol)-co-polystyrene polymersomes in which particles formed due to aggregation at $55^{\circ} \mathrm{C},{ }^{44}$ and changes in morphology of poly(ethyleneglycol)-block-poly[(2-phenylboronate esters-1,3-dioxane5ethyl)methylacrylate] and poly(dimethylsiloxane)-g-poly(ethylene oxide) polymersomes in response to glucose and hypertonic shock, respectively. ${ }^{45,46}$

Thus, despite an increasing interest in temperature-responsive self-assembled statures including our recent study on synthesis and drug delivery potential of $\mathrm{PVCL}_{\mathrm{n}}-\mathrm{PDMS}_{\mathrm{m}^{-}}$ $\mathrm{PVCL}_{n}$ polymersomes, ${ }^{31}$ there is lack of understanding of their assembly mechanism. The molecular-level picture of their temperature-induced structural changes has been also overlooked, which significantly limits their applications for controlled delivery. To address these issues, we investigate dimensional changes within $\mathrm{PVCL}_{\mathrm{n}}-\mathrm{PDMS}_{\mathrm{m}}-\mathrm{PVCL}_{\mathrm{n}}$ polymersome membranes at physiologically relevant temperatures from 25 to $55^{\circ} \mathrm{C}$ and compare the effects of polymer type, molecular weight, and temperature on the structure of the polymersomes using SANS, TEM, light scattering, and all-atom molecular dynamics (MD) simulation. We also explore how the weight ratio of hydrophilic PVCL blocks to the overall molecular weight $f$ is related to the changes in the polymersome structure at these temperatures. Our SANS data demonstrate that the low and high hydrophilic ratios result in to two distinct types of hydrophilic/hydrophobic transitions within the inner and outer membrane of the polymersome which can lead to a different permeability behavior of these synthetic vesicles. All-atom MD simulations are used to investigate temperature-induced conformational transitions of individual hydrophilic blocks. Our study on the behavior of temperature-responsive polymersome shells can significantly advance the field of temperature-responsive synthetic polymersomes for controlled delivery of functional molecules.

\section{Materials and Methods}

2,2' - Azobis(2-methylpropionitrile) (AIBN) (Sigma-Aldrich) was purified by recrystallization from methanol prior to use. N-Vinylcaprolactam (VCL) (Sigma-Aldrich) and tetrahydrofuran (Fisher) was distilled before reaction. Potassium ethyl xanthate, 2bromopropionyl bromide, diethyl ether, hexane, dichloromethane, calcium sulfate anhydrous, and dialysis tubing (MWCO $5 \mathrm{kDa}$ ) (Fisher) were used as received. 
Bis(hydroxyalkyl) poly(dimethylsiloxane) $\left(\mathrm{PDMS}_{65}, \mathrm{Mn}=5600 \mathrm{Da}\right)$ was purchased from Sigma-Aldrich. Triflic acid was purchased from Sigma-Aldrich.

\section{Synthesis of temperature-responsive $\mathrm{PVCL}_{n}-\mathrm{PDMS}_{65}-\mathrm{PVCL}_{n}$ triblock copolymers.}

As previously reported, poly(N-vinylcaprolactam) $)_{n}$-poly(dimethylsiloxane) ${ }_{65}$-poly(N-vinyl-

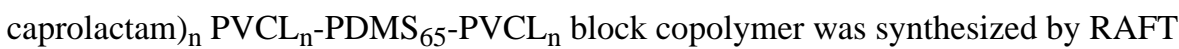
(Reversible Addition-Fragmentation chain Transfer) polymerization. ${ }^{31}$ In brief, bis(hydroxyalkyl) poly(dimethylsiloxane) $\left(\mathrm{PDMS}_{65}\right)$ was first modified with 2bromopropionyl bromide followed by potassium ethyl xanthogenate resulting in the PDMS macro-initiator. $\mathrm{PVCL}_{10}-\mathrm{PDMS}_{65}-\mathrm{PVCL}_{10} \mathrm{PVCL}_{15}-\mathrm{PDMS}_{65}-\mathrm{PVCL}_{15}$, and $\mathrm{PVCL}_{21^{-}}$ $\mathrm{PDMS}_{65}-\mathrm{PVCL}_{21}$ were synthesized after RAFT living radical polymerization of VCL monomer by controlling the reaction time with a feed ratio of 1:1:80 by weight of PDMSinitiator/AIBN/monomer for 4 hours, 6 hours and 10 hours, respectively. Monomer conversion and purified molecular weight of the copolymer were determined by ${ }^{1} \mathrm{H}$ NMR. The number-average molecular weights and polydispersity of triblock copolymers were obtained using gel permeation chromatography (GPC) analysis (Table S1; Figure S1). GPC was performed on a Waters system equipped with a Waters 1515 pump, a Waters 2414 differential refractive index detector, and two connected Styragel columns. Tetrahydrofuran was used as eluent with $0.02 \mathrm{M}$ tetra-n-butylammonium fluoride trihydrate (TBAF) at the flow of $1 \mathrm{~mL}$ min-1 at room temperature. The calibration was performed with monodisperse linear polystyrene standards (Waters).

\section{Synthesis of non-temperature-responsive Poly-(2-methyl-2-oxazoline) ${ }_{14}$-Poly (dimethylsiloxane) 65 -Poly-(2-methyl-2-oxazoline) ${ }_{14}$ (PMOXA $14_{14}-$ PDMS $_{65}-$ PMOXA $\left._{14}\right)$ triblock polymers.}

Poly-(2-methyl-2-oxazoline) ${ }_{14}$-poly(dimethylsiloxane) ${ }_{65}$ poly-(2-methyl-2-oxazoline) 14 block copolymer was synthesized by anion polymerization. In brief, bis(hydroxyalkyl) poly(dimethylsiloxane) $\left(\mathrm{PDMS}_{65}\right)$ was first modified with triflate to produce the PDMS macro-initiator. $\mathrm{PMOXA}_{14}-\mathrm{PDMS}_{65}-\mathrm{PMOXA}_{14}$ was synthesized by anion polymerization with 2-methyl-2-oxazoline using a reaction time of 2.5 hours.

\section{Assembly of PVCL $-\mathrm{PDMS}_{65}-\mathrm{PVCL}_{n}$ and $\mathrm{PMOXA}_{14}-\mathrm{PDMS}_{65}-\mathrm{PMOXA}_{14}$ Polymersomes.}

The vesicles were prepared using nanoprecipitation or thin film hydration methods. Briefly, for nanoprecipitation, $1 \mathrm{~mL}$ of an ethanol solution of an ABA block copolymer at $5 \mathrm{mg} \mathrm{mL}$

${ }^{-1}$ was added dropwise to $5 \mathrm{~mL}$ of deionized (DI) water at room temperature and stirred for $6 \mathrm{~h}$, followed by dialysis of the mixture in water for $24 \mathrm{~h}$ to remove ethanol. To prepare polymersomes using a thin film hydration method, a thin film of copolymer was formed by slow evaporation of the solvent from $0.5 \mathrm{mg} \mathrm{mL}^{-1}$ block copolymer solutions in $\mathrm{CH}_{2} \mathrm{Cl}_{2}$ using a rotary evaporator. The thin copolymer films were hydrated in DI water or $\mathrm{D}_{2} \mathrm{O}$ for $24 \mathrm{~h}$ under stirring followed by a double extrusion through a $0.2-\mu \mathrm{m}$ pore size polycarbonate membrane using an Avanti mini-extruder (Avanti Polar Lipids, Incorporated). 


\section{Dynamic Light Scattering (DLS).}

The vesicle size was measured using a Nano-ZS Zetasizer (Malvern) equipped with a He $-\mathrm{Ne}$ laser $(663 \mathrm{~nm})$. The average diameter of the assembled particles was obtained from three independent runs (15 measurements each). The particle size distribution was evaluated by standard deviation values.

\section{Small-Angle Neutron Scattering (SANS).}

SANS experiments were carried out with the Bio-SANS instrument at the High-Flux Isotope Reactor (HFIR) at Oak Ridge National Laboratory. A constant neutron wavelength of $6 \AA$ with a relative wavelength spread of $\sim 15 \%$ was used in all measurements. One single instrument configuration was able to cover the q range of 0.003 to $0.7 \AA^{-1}$ by utilizing a combination of a main and a wing detector. The scattering intensity profiles $\mathrm{I}(\mathrm{q})$ versus $\mathrm{q}$ were obtained by azimuthally averaging the processed $2 \mathrm{D}$ images, which were normalized to neutron flux monitor, and scaled by using water as a secondary standard, and corrected for detector dark current, pixel sensitivity, sample transmission, and scattering from backgrounds $\left(\mathrm{D}_{2} \mathrm{O}\right.$ and quartz cell). The SANS data were reduced with the facility-provided data reduction software Mantid. The polymersomes were suspended in $\mathrm{D}_{2} \mathrm{O}$ before being placed in quartz cuvettes (Hellma, Germany) of $2 \mathrm{~mm}$ beam path for SANS measurements. The sample was mounted on the metal sample holder with a titanium spacer. The temperature was tuned with a circulation water bath which pumped hot water through the holder. NIST SANS data analysis packages in Igor $\mathrm{Pro}^{47}$ were used for data analysis. Fit quality was determined by minimizing the goodness-of-fit parameter $\left(\chi^{2}\right)$, and parameters were constrained to physically reasonable values of size and thickness and consistency with other measurements, such as DLS and TEM.

The data was quantitatively analyzed by performing modified Guinier analysis. At larger q, corresponding to smaller length scales, where the thickness is much smaller than the overall size of the polymersome, the curvature of the polymersome becomes unimportant; instead it can be viewed as a sheet with a thickness $\mathrm{d}$. For $\mathrm{qRg}<1$, where $\mathrm{Rg}$ is related to the thickness as $d=(12)^{1 / 2} \operatorname{Rg}$, the intensity of scattering from a sheet goes as

$$
\mathrm{I}(\mathrm{q}) \propto \frac{1}{q^{2}} e^{-q^{2} g^{2}}
$$

Therefore, from fitting $\ln \left(\mathrm{I}(\mathrm{q}) \mathrm{q}^{2}\right)$ versus $\mathrm{q}^{2},-\mathrm{Rg}^{2}$ can be derived from the slope from which the thickness can be calculated.

The Guinier_Porod model was applied to obtain the overall size, $\operatorname{Rg}_{1}$ of the polymersomes. The scattering intensity $\mathrm{I}(\mathrm{q})$ is given as

$$
\mathrm{I}(\mathrm{q})=\mathrm{I}_{0} e^{-\frac{q^{2} R g 1^{2}}{3-s}}
$$

where $\mathrm{q}=(4 \pi / \lambda) \sin (\theta / 2), \lambda$ is the neutron wavelength, $\theta$ is the scattering angle. $\operatorname{Rg}_{1}$ is the radius of gyration of the particles, and $\mathrm{s}$ is the dimension variance. 
The core-shell model was used to fit the data to obtain parameters on the detailed structure of the polymersome shell. The form factor $\mathrm{P}(\mathrm{q})$ is normalized by the mean particle volume such that

$$
P(q)=\frac{s c a l e}{V_{s}}\left[\frac{3 V_{s}\left(\rho_{c}-\rho_{s}\right) j_{1}\left(q r_{c}\right)}{q r_{c}}+\frac{3 V_{s}\left(\rho_{c}-\rho_{\text {solv }}\right) j_{1}\left(q r_{s}\right)}{q r_{s}}\right]^{2}+b k g
$$

where $j_{1}(x)=(\sin x-x \cos x) / x^{2}$ is the second spherical Bessel function, $\rho_{\mathrm{c}}$ is $\operatorname{SLD}$ (scattering length density) of the core, $\rho_{\mathrm{s}}$ is SLD of the shell and $\rho_{\text {solv }}$ is SLD of the solvent. The returned form factor is normalized by the average particle volume $\mathrm{v}=\frac{4 \pi r^{3}}{3}$ where $\mathrm{r}$ denotes the total particle radius: $\mathrm{r}=\mathrm{Rc}+\mathrm{t}$, where $\mathrm{Rc}$ is radius of the core, $\mathrm{t}$ is thickness of the shell. Fit parameters obtained from the poly core-shell model deduced from SANS curves are presented in Table S2.

\section{Transmission Electron Microscopy (TEM).}

TEM images of polymersomes were obtained with FEI Tecnai T12 Spirit TWIN TEM microscope operated at $80 \mathrm{kV}$. $7 \mu \mathrm{L}$ of a vesicle solution was dropped onto an argon plasmatreated Formvar/carbon coated copper grid (200 mesh, HRIF). The deposited polymersomes were stained with $1 \%(\mathrm{wt})$ uranyl acetate for 30 seconds after rinsing off excess solution. For cryogenic electron microcopy (cryo-TEM), $3 \mu \mathrm{L}$ of sample was applied to glow-discharged 200 mesh Quantifoil R 2/1 grids (Electron Microscopy Sciences, Hatfield, PA). The grid was loaded into the FEI Vitrobot Mark IV (FEI, Eindhoven, Netherlands), where it was blotted briefly and plunged into liquid ethane. Frozen grids were transferred to a Gatan 622 cryoholder and observed in an FEI Tecnai F20 electron microscope (Eindhoven, Netherlands) operated at $200 \mathrm{kV}$ with magnification at $65500 \times$ and defocus settings of -2.5 to $-3.0 \mu \mathrm{m}$. Images were collected under low-dose conditions on a Gatan Ultrascan 4000 CCD camera.

\section{Molecular Dynamics (MD) simulations.}

All simulations were performed using the classical MD package NAMD, ${ }^{48}$ periodic boundary conditions, and a $2 \mathrm{fs}$ integration time step. The CHARMM 36 force field ${ }^{49}$ was used to describe polymer, TIP3P water, and ions along with the CUFIX corrections applied to improve description of charge-charge interactions. ${ }^{50}$ RATTLE $^{51}$ and SETTLE $^{52}$ algorithms were applied to covalent bonds that involved hydrogen atoms in polymer and water molecules, respectively. The particle mesh Ewald (PME) ${ }^{53}$ algorithm was adopted to evaluate the long-range electrostatic interaction over a $1 \AA$-spaced grid. Van der Waals interactions were evaluated using a smooth $10-12 \AA$ cutoff. Multiple time stepping was used to calculate local interactions every time step and full electrostatics every three time steps. The Nose-Hoover Langevin piston pressure control $^{54}$ was used to maintain the pressure of the system at $1 \mathrm{~atm}$ by adjusting the system's dimension. Langevin thermostat ${ }^{55}$ was applied to all the heavy atoms of the system with a damping coefficient of $0.1 \mathrm{ps}^{-1}$ to maintain the system temperature at either $275,295,315$ or $328 \mathrm{~K}$.

The PVCL systems were created by first using MarvinSketch ${ }^{56}$ to draw the chemical structures of the polymers. The chemical structure files were converted to all-atom 
representation using the molecule editor software Avogadro. ${ }^{57}$ The structure files were also used to obtain CHARMM36-compatible parameters for the PVCL polymers using the CgenFF online tool. To build a typical simulation system, one PVCL polymer was solvated in a rectangular volume of TIP3P water molecules. Potassium and chloride ions were added to produce a $0.4 \mathrm{M}$ electrolyte solution. Each PVCL systems measured approximately $4 \times 4$ $\times 4 \mathrm{~nm}^{3}$ and contained approximately 5,800 atoms. Following assembly, each system was energy-minimized for 6000 steps using the conjugate gradient method and then equilibrated for $10 \mathrm{~ns}$ in the NPT ensemble at $295 \mathrm{~K}$. Following that, each system was simulated at 275, 295,315 and $328 \mathrm{~K}$ for $320 \mathrm{~ns}$. Three additional replica simulations were carried out at each temperature lasting from 120 to $320 \mathrm{~ns}$ each.

\section{Results and Discussion}

\section{Synthesis of Thermoresponsive and Non-Thermoresponsive Triblock Copolymers}

All PVCL-PDMS-PVCL triblock copolymers were obtained via polymerization of hydrophilic monomers onto both sides of a PDMS block modified with a xanthate chain transfer agent (CTA). ${ }^{31}$ Bis(hydroxyethyl)oxypropyl poly(dimethylsiloxane) was prepared by condensation reaction of dimethoxydimethylsilane with 1,3-bis(4hydroxybutyl)tetramethyldisiloxane to obtain hydroxy-alkyl terminated end groups on both sides. In these preparations, a methanol/water extraction removed lower molecular weight fractions, including cyclics, and thus yielded a more uniform product with a narrow molecular weight distribution. The number-average molecular weight of bis(hydroxyethyl)oxypropyl poly(dimethylsiloxane) was calculated from NMR analysis based on the ratio between the integrals from $\delta=0$ to $\delta=0.3 \mathrm{ppm}\left(\mathrm{SiCH}_{3}\right.$ protons in the PDMS block) and at $\delta=0.6 \mathrm{ppm}$ (the methylene protons in the end groups) (Figure S2). The macro initiator XPDMS-X was prepared by converting the hydroxy-terminated PDMS into the intermediate Br-PDMS-Br (Figure S1) via reaction with 2-bromopropionyl bromide, followed by a subsequent reaction with the CTA potassium ethyl xanthogenate. ${ }^{31}$ The NMR analysis revealed that $97 \%$ of the PDMS hydroxy groups were modified by the CTA (Figure S2). Due to the high extent of conversion, the likelihood of formation of unwanted diblock copolymers (single end-reacted CTA) was very low, which translates to both control over the reaction rates through the CTA at both ends of the macroinitiator and ensemble behavior that is effectively representative of only triblock copolymers.

To compare polymersomes having thicker and thinner membranes and study the effects of hydrophilic weight ratios on longer and shorter polymers, we used PDMS $30, \mathrm{PDMS}_{47}$, and $\mathrm{PDMS}_{65}$ as macroinitiators to prepare the PVCL-based temperature responsive copolymers. RAFT polymerizations of $\mathrm{N}$-vinylcaprolactam $(\mathrm{VCL})$ using $\mathrm{X}-\mathrm{PDMS}_{65}-\mathrm{X}$ with the specific reaction time of 6,8 , and 12 hours yielded $\mathrm{PVCL}_{\mathrm{n}}-\mathrm{PDMS}_{65}-\mathrm{PVCL}_{\mathrm{n}}$ where $\mathrm{n}=10,15$, and 21, respectively, while 3- and 4-hour reactions were used to prepare $\mathrm{PVCL}_{5}-\mathrm{PDMS}_{30^{-}}$ $\mathrm{PVCL}_{5}$ and $\mathrm{PVCL}_{7}-\mathrm{PDMS}_{47}-\mathrm{PVCL}_{7}$, respectively. The PVCL block length was determined by comparing the integral at $\delta=2.5 \mathrm{ppm}$ (methylene protons next to the carbonyl on each PVCL repeat unit) to the integral from $\delta=0 \mathrm{ppm}$ to $\delta=0.3 \mathrm{ppm}\left(\mathrm{SiCH}_{3}\right.$ protons in the PDMS block) (Figure 1(a)). The length of a single PVCL block was calculated as half of the total PVCL units. Importantly, the block lengths of the copolymers in this study were guided 
by the weight ratio $f$ of hydrophilic monomer units to the total polymer weight within the range of 0.25 to 0.4 , with spherical micelles formed when this ratio is larger than 0.5. ${ }^{31}$ Indeed, both $\mathrm{PVCL}_{10}-\mathrm{PDMS}_{65}-\mathrm{PVCL}_{10}(f=0.36)$ and $\mathrm{PVCL}_{15}-\mathrm{PDMS}_{65}-\mathrm{PVCL}_{15}(f=0.46)$ assembled into vesicles; however, we observed that $\mathrm{PVCL}_{21}-\mathrm{PDMS}_{65}-\mathrm{PVCL}_{21}(f=0.54)$ was also able to form vesicles despite its relatively high $f$ value. Our previous study has confirmed that ratios larger than this $(f>0.62)$ indeed produce micellar structures rather than polymersomes. ${ }^{31}$ Interestingly, in the case of $\mathrm{PDMS}_{30}$, for achieving $f$ ratios between 0.3 and 0.5 , the PVCL content was limited to the range between 5 and 10 repeat units on each end. This would be a particular challenge to the synthetic methodology had it not been for the reaction rate limiting CTA on both sides of the $\mathrm{X}-\mathrm{PDMS}_{30} \mathrm{X}$ macroinitiator.

Polymersomes with no temperature response were assembled from triblock copolymers of poly(2-methyl-2-oxazoline) ${ }_{14}$-poly(dimethylsiloxane) 65 -poly(2-methyl-2-oxazoline) 14 $\left(\mathrm{PMOXA}_{14}-\mathrm{PDMS}_{65}-\mathrm{PMOXA}_{14}\right.$ ) which were synthesized by anionic polymerization of 2methyl-2-oxazoline (MOXA) using triflic acid activated $a, \omega$-bis(3-hydroxypropyl) poly(dimethylsiloxane) according to a previously reported procedure. ${ }^{58}$ The chain length of PMOXA was controlled by the reaction time, with 2.5 hours yielding 14 MOXA units at both ends of the PDMS. The block ratios were calculated from NMR analysis, comparing the ratio between the integrals from $\delta=0$ to $\delta=0.3 \mathrm{ppm}\left(\mathrm{SiCH}_{3}\right.$ protons in the PDMS block) and at $\delta=0.6 \mathrm{ppm}$ (the methylene protons in the end groups) to integrals at $\delta=2.1$ ppm belonging to the methyl group of MOXA (Figure 1(b)). The repeat unit length of 14 was chosen to target an $f$ value similar to the $f$ range of the synthesized PVCL-PDMS-PVCL copolymers. PMOXA does not exhibit the temperature responsive behavior and has been used as the hydrophilic block of other polymersome architectures and was therefore useful as a control in our temperature-dependent DLS and SANS experiments.

\section{Self-Assembly of Temperature-Responsive and Non-Temperature Responsive Polymer Vesicles}

Temperature-responsive and non-temperature responsive polymersomes were assembled from PVCL-PDMS-PVCL and PMOXA-PDMS-PMOXA copolymers, respectively, using the thin film rehydration method with rehydration in $\mathrm{D}_{2} \mathrm{O}$ (see Experimental) and extrusion through a $0.2-\mu \mathrm{m}$ pore filter. Figure $2(\mathrm{a}-\mathrm{f})$ shows TEM images of vesicles made from $\mathrm{PVCL}_{10}-\mathrm{PDMS}_{65}-\mathrm{PVCL}_{10}, \mathrm{PMOXA}_{14}-\mathrm{PDMS}_{65}-\mathrm{PMOXA}_{14}, \mathrm{PVCL}_{15}-\mathrm{PDMS}_{65}-\mathrm{PVCL}_{15}$, $\mathrm{PVCL}_{21}-\mathrm{PDMS}_{65}-\mathrm{PVCL}_{21}, \mathrm{PVCL}_{7}-\mathrm{PDMS}_{47}-\mathrm{PVCL}_{7}$ and $\mathrm{PVCL}_{5}-\mathrm{PDMS}_{30}-\mathrm{PVCL}_{5}$, respectively, after extrusion. The images show that all of the block copolymers produced vesicular morphologies upon self-assembly with a small size distribution. The nonpermeability of the uranyl acetate stain evidences that the membrane remained intact and illustrates the spherical morphology of the polymersomes. The average polymersome diameter at room temperature was 390, 218, and $186 \mathrm{~nm}$ for $\mathrm{PVCL}_{\mathrm{n}}-\mathrm{PDMS}_{65}-\mathrm{PVCL}_{\mathrm{n}}$ polymersomes with PVCL $\mathrm{n}=10,15$, and 21 , respectively, while polymersomes of $\mathrm{PVCL}_{5^{-}}$ $\mathrm{PDMS}_{30}-\mathrm{PVCL}_{5}$ and $\mathrm{PVCL}_{7}-\mathrm{PDMS}_{47}-\mathrm{PVCL}_{7}$ had average sizes of 280 and $299 \mathrm{~nm}$, respectively, as measured by DLS (Table 1). As is seen, increasing hydrophilic (PVCL) content decreases the vesicle size due to an increase in the hydrophilic corona steric repulsion as reported previously. ${ }^{31,59}$ Thus, the much shorter $\mathrm{PVCL}_{5}-\mathrm{PDMS}_{30}-\mathrm{PVCL}_{5}$ copolymer forms polymersomes that are $\sim 1.5$ times larger than those of the longer $\mathrm{PVCL}_{21^{-}}$ 
$\mathrm{PDMS}_{65}-\mathrm{PVCL}_{21}$ copolymer. It is clear from these observations that the hydrophilic weight ratio $f$ of the copolymer plays the most significant role in determining the size of the selfassembled vesicle. It should be noted that our previous work showed polymersomes formed from $\mathrm{PVCL}_{\mathrm{n}}-\mathrm{PDMS}_{65}-\mathrm{PVCL}_{\mathrm{n}}$ with PVCL $\mathrm{n}=10$ and 15 had an average hydrodynamic size of 530 and $220 \mathrm{~nm}$, respectively, when prepared using the nanoprecipitation method and were stable for $72 \mathrm{~h}$ as measured by DLS. ${ }^{31}$ The polymersomes in the current study obtained via thin film rehydration were smaller than those produced by nanoprecipitation, which can be explained by the fact that our thin film rehydration vesicles were forced through the small pores of a membrane during extrusion. As the polymersomes are soft, fluid-like vesicles, the self-assembly conditions and subsequent manipulations serve to physically influence the vesicle size.

\section{Temperature-Induced Dimensional Changes in PVCL-PDMS-PVCL Polymersomes}

The PVCL-PDMS-PVCL polymersomes exhibit dynamic temperature-dependent size changes due to the LCST behavior of PVCL. ${ }^{31}$ The diameter of the polymersomes at $25{ }^{\circ} \mathrm{C}$ (below the phase transition temperature) and $55{ }^{\circ} \mathrm{C}$ (above the phase transition temperature) was therefore explored with DLS. Figure 3(a) and (b) shows the average hydrodynamic sizes of $\mathrm{PVCL}_{10}-\mathrm{PDMS}_{65}-\mathrm{PVCL}_{10}$ and $\mathrm{PMOXA}_{14}-\mathrm{PDMS}_{65}-\mathrm{PMOXA}_{14}$ at both temperatures. The polymersomes formed from $\mathrm{PVCL}_{10}-\mathrm{PDMS}_{65}-\mathrm{PVCL}_{10}$ demonstrated a $20 \%$ decrease in size (from 390 to $315 \mathrm{~nm}$ ) when the temperature changed from 25 to $55^{\circ} \mathrm{C}$, while the PMOXA-based polymersomes exhibited no change in diameter. In Figure 3(c) and (d), the size of the $\mathrm{PVCL}_{15}-\mathrm{PDMS}_{65}-\mathrm{PVCL}_{15}$ and $\mathrm{PVCL}_{21}-\mathrm{PDMS}_{65}-\mathrm{PVCL}_{21}$ polymersomes is shown via DLS plots at 25 and $55^{\circ} \mathrm{C}$. Upon elevation of the solution temperature, the average hydrodynamic sizes of $\mathrm{PVCL}_{15}-\mathrm{PDMS}_{65}-\mathrm{PVCL}_{15}$ and $\mathrm{PVCL}_{21}-\mathrm{PDMS}_{65}-\mathrm{PVCL}_{21}$ polymersomes decreased from 218 to $178 \mathrm{~nm}$ and from 186 to $171 \mathrm{~nm}$, respectively, representing 18 and $8 \%$ changes in size, respectively. The data shows that polymersomes with higher $f$ ratios exhibit both a smaller initial size at $25{ }^{\circ} \mathrm{C}$ and lower extent of shrinkage at $55^{\circ} \mathrm{C}$. The longer PVCL segments are known to result in smaller vesicle diameters due to the additive intermolecular repulsion between neighboring hydrophilic chain segments attached to and extending out from the surface of the membrane that forms during selfassembly, as the contiguous hydrophobic PDMS membrane responsible for stable vesicle formation is more favorably compacted than stretched in a hydrophilic solvent. ${ }^{9}$ The smaller vesicle diameter and increased chain length results in PVCL chains that pack more densely within the solvated portion of the polymersome corona. In this case, the shedding of solvating water molecules at the elevated temperature would be expected to have a smaller effect on the size of the vesicle since the longer chains extending inward from the vesicle surfaces have less free volume in which to collapse. In essence, the already decreased size at ambient temperature occurring from longer chain segments serves to preempt the ability of the vesicles to decrease much further in size during the LCST transition of the hydrophilic segments due to the elevated chain density on the inner and outer surface of the membrane.

Figure 3(e) and (f) shows DLS measurements of $\mathrm{PVCL}_{7}-\mathrm{PDMS}_{47}-\mathrm{PVCL}_{7}$ and $\mathrm{PVCL}_{5}$ $\mathrm{PDMS}_{30}-\mathrm{PVCL}_{5}$ polymersomes. For these shorter copolymers, the size changes were similar as the lengths of the PVCL segments differ only by two units. The PVCL5-PDMS $30^{-}$ $\mathrm{PVCL}_{5}$ polymersomes changed from 280 to $215 \mathrm{~nm}$ in diameter while those from $\mathrm{PVCL}_{7}$ - 
$\mathrm{PDMS}_{47}-\mathrm{PVCL}_{7}$ changed in size from 299 to $245 \mathrm{~nm}$ when the solution temperature increased from 25 to $55^{\circ} \mathrm{C}$. This change of $\sim 25$ and $18 \%$, respectively, agrees well with the temperature responsiveness of $\mathrm{PVCL}_{10}-\mathrm{PDMS}_{65}-\mathrm{PVCL}_{10}$ polymersomes in which the size change was $\sim 20 \%$. This data also supports the trend of a large diameter change with lower $f$ ratio as the $\mathrm{PVCL}_{5}-\mathrm{PDMS}_{30}-\mathrm{PVCL}_{5}(f=0.33)$ and $\mathrm{PVCL}_{7}-\mathrm{PDMS}_{47}-\mathrm{PVCL}_{7}(f=0.36)$ both fall at the lower end of the $f$ range at which polymersomes are expected to form.

It should be noted that all DLS measurements were carried out in $\mathrm{D}_{2} \mathrm{O}$ to maintain consistency with the results obtained from the SANS experiments. Interestingly, the size shrinkage of the polymersomes $(20 \%)$ at $55^{\circ} \mathrm{C}$ is lower than we previously reported (30\%), which may be attributed to hydrogen bonding in $\mathrm{D}_{2} \mathrm{O}$ that requires more energy to disrupt due to the doubled atomic mass of deuterium compared to protium. ${ }^{60}$ Indeed, it has also been shown that the $\Delta \mathrm{H}$ of chain collapse for PNIPAM is higher in $\mathrm{D}_{2} \mathrm{O}$ than in water. ${ }^{61}$ Since it has been demonstrated that hydrogen bonding between water and PVCL becomes less favorable when the solution temperature is higher than the LCST, ${ }^{62}$ the stronger comparative hydrogen bonding and higher viscosity of $\mathrm{D}_{2} \mathrm{O}^{63}$ at the same temperature may affect the extent of the LCST transition. We tested this hypothesis by determining the cloud point of solutions of PVCL homopolymer in $\mathrm{H}_{2} \mathrm{O}$ and $\mathrm{D}_{2} \mathrm{O}$ using fluorometry (Figure $\mathrm{S}$ ) to compare the phase transition temperatures. At the concentration of $0.5 \mathrm{mg} \mathrm{mL}^{-1}$, the solution of PVCL in $\mathrm{H}_{2} \mathrm{O}$ exhibited phase transition onset at $32.4^{\circ} \mathrm{C}$ while that of the $\mathrm{D}_{2} \mathrm{O}$ solution was $33.6^{\circ} \mathrm{C}$. This slight increase in the transition temperature is in agreement with the study mentioned above showing an increase in the LCST of PNIPAM by $0.6{ }^{\circ} \mathrm{C} .61$

\section{Temperature-Induced Size and Thickness Changes in PVCL-PDMS-PVCL Polymersomes studied by SANS}

We previously showed that temperature-responsive polymersomes can release the model drug DOX in response to their LCST-mediated change in size. ${ }^{31}$ However, it would be beneficial to the development of temperature-responsive polymersomes in general to gain a thorough understanding of the mechanism through which small molecules permeate the hydrophobic membrane. For this reason, SANS was employed to characterize the changes in polymersome shell thickness and structure in solution above and below the LCST of the hydrophilic polymer block. The size of the $\mathrm{PVCL}_{10}-\mathrm{PDMS}_{65}-\mathrm{PVCL}_{10}$ polymersomes was evaluated with SANS to compare to the DLS data (Figure 4 (a)). The Guinier-Porod model can be used to calculate $\mathrm{R}_{\mathrm{g}}$, dimension variance, and the Porod exponent which describes the particle shape and surface fractals. ${ }^{64}$ For the polymersomes in this study, analysis of the model fit resulted in a dimension variance of $6.9 \times 10^{-7}$, which being very close to zero indicates that the vesicles have a $3 \mathrm{D}$ globule shape. At the higher q range where Porod exponents dictate the shape of the scattering curve, the Porod exponent is 3.846. According to the model, a Porod exponent of 4 indicates the surface of a smooth sphere. The slightly lower Porod exponent in our study indicates surface fractalization or roughness that is likely due to the presence of randomly coiled polymer chains extending from the inner and outer surfaces of the membrane. The model fit predicted $R_{g}$ of $170 \mathrm{~nm}$, which being slightly lower than the hydrodynamic radius $\left(\mathrm{R}_{\mathrm{h}}\right)$ calculated by DLS $(390 \mathrm{~nm} / 2=185 \mathrm{~nm})$ agrees well with a physical representation in between that of a solid sphere $\left(\mathrm{R}_{\mathrm{g}}{ }^{2}=3 \mathrm{r}^{2} / 5\right)$ and a hollow sphere with an infinitesimally thin shell $\left(R_{g}=r\right)$. As the $R_{h}$ calculated by DLS is the 
diameter of an equivalent hard sphere scattering at the same correlation as the predicted particle size, a slightly lower calculated $\mathrm{R}_{\mathrm{g}}$ describes a relatively hollow sphere with polymer chains extending from either surface of a membrane having non-negligible thickness.

For our triblock copolymer vesicles, the hydrophobic PDMS blocks constitute the majority of the vesicle wall, while the chains of water soluble PVCL blocks extend from the inner and outer surfaces into the solvent system. Since the samples were prepared in $100 \% \mathrm{D}_{2} \mathrm{O}$, the hydrophobic region formed by PDMS, besides contributing to the majority of the shell thickness, also contributed the greater portion of the SLD contrast due to near-absolute rejection of solvating $\mathrm{D}_{2} \mathrm{O}$ molecules. However, for the shell region formed from PVCL segments, the polymer chains were much more hydrated by $\mathrm{D}_{2} \mathrm{O}$ and, while contributing a small degree to the thickness, also had limited contrast against the solvent. Importantly, the thickness contributed by the hydrophilic region should not be neglected during the measurement of the shell thickness, as it plays a significant role in the stability and dynamics of the self-assembled system and in our case is responsible for the temperature-responsive behavior of the polymersomes. As the core-shell model represents the ensemble average SLD of the polymersome shells in suspension, the fit parameter was constrained between the SLD of the solvent and the calculated value of PDMS to average the SLD of the hydrated and insoluble regions of the shell and include the contributions from both segments of the copolymer in the scattering model.

The thickness of the $\mathrm{PVCL}_{10}-\mathrm{PDMS}_{65}-\mathrm{PVCL}_{10}$ polymersome membranes was explored by utilizing analysis from model fitting of the scattering curves and the model-free modified Guinier plot ${ }^{65}$ in Figure 4(a) and (b). In the modified Guinier plot, when the q range is limited to $\mathrm{qR}_{\mathrm{g}}<1$, the thickness is related to the $\mathrm{R}_{\mathrm{g}}$ as $\mathrm{d}=(12)^{1 / 2} \mathrm{R}_{\mathrm{g}}$ and the slope of a linear fit to the data is equal to $-\mathrm{R}_{\mathrm{g}}{ }^{2}$. In our case, for $\mathrm{q}<0.032 \AA^{-1}$, a linear fit to the data at $25^{\circ} \mathrm{C}$ gives a slope of -1773.4 , from which the thickness of the $\mathrm{PVCL}_{10}-\mathrm{PDMS}_{65}-\mathrm{PVCL}_{10}$ polymersome membrane was calculated to be $14.5 \mathrm{~nm}$ (Figure 4(b)). The core-shell model was then applied to the same scattering curve to extract parameters for the polymersome diameter and shell thickness together, while also elucidating the SLD of the shell and core of the polymersomes. The fits to the core-shell model for $\mathrm{PVCL}_{10}-\mathrm{PDMS}_{65}-\mathrm{PVCL}_{10}$ vesicles at 25 and $55^{\circ} \mathrm{C}$ are shown in Figure 4(c). The sample was diluted by a factor of 10 during the high temperature measurement to prevent possible aggregation, and the scaling parameter was adjusted to balance the effect of the concentration during the model fitting. Extracted size and shell thickness for the model fits at $25^{\circ} \mathrm{C}$ gave a size of $352 \mathrm{~nm}$ and thickness of $14.1 \mathrm{~nm}$, while the size and thickness decreased to 286 and $11.7 \mathrm{~nm}$, respectively at $55{ }^{\circ} \mathrm{C}$. At $25{ }^{\circ} \mathrm{C}$, the size given by the core-shell model agrees with the $\mathrm{R}_{\mathrm{h}}$ obtained from DLS (Figure 3(a)) and the $\mathrm{R}_{\mathrm{g}}$ calculated from the Guinier-Porod model (Figure 4(a)). The cryo-TEM analysis of $\mathrm{PVCL}_{10}-\mathrm{PDMS}_{65}-\mathrm{PVCL}_{10}$ polymersomes resulted in the polymersome shell thickness of $14.6 \pm 0.4 \mathrm{~nm}$ (Figure $\mathrm{S} 4$ (a)) which is in good agreement with the SANS data discussed above. The membrane thickness is also in excellent agreement with the result from the model-free modified Guinier plot (Figure 4(b)), indicating the accuracy and validity of the core-shell model to describe the characteristics of these polymersomes. At $55^{\circ} \mathrm{C}$, the model fit parameters indicated that the $\mathrm{PVCL}_{10^{-}}$ $\mathrm{PDMS}_{65}-\mathrm{PVCL}_{10}$ polymersome diameter decreased around $25 \%$, which corroborates the 
DLS results. Interestingly, a decrease in shell thickness of $\sim 17 \%$ was also found through model fitting of the SANS data. This can be explained by the interruption of hydrogen bonding between PVCL and $\mathrm{D}_{2} \mathrm{O}$ and the enhanced hydrophobic interactions between PVCL segments at high temperature that cause a coil-globule transition of the PVCL chains. ${ }^{66}$ We have also recently shown through neutron reflectometry ${ }^{67}$ that the expulsion of $\mathrm{D}_{2} \mathrm{O}$ from hydrogel networks at high temperature resulting from the reversible volume phase transition of PVCL chains causes the network to collapse and results in a significant decrease in the thickness of assembled films. Furthermore, in the case of our $\mathrm{PVCL}_{10^{-}}$ $\mathrm{PDMS}_{65}-\mathrm{PVCL}_{10}$ polymersomes, the hydrophilic PVCL blocks on the inner and outer surface of the membrane are relatively short, comprising not many more units than the persistence length of the polymer. ${ }^{68}$ At room temperature, the PDMS blocks are pulled in both directions by the hydration-induced swelling of the PVCL blocks. However, at elevated temperature, the enhanced hydrophobicity of the PVCL blocks can result in an exaggerated collapse of the whole copolymer and a decrease in the thickness of the polymersome membrane ensues. As will be discussed later, this data reinforces the importance of the length of the hydrophilic blocks and $f$ ratio in terms of both the behavior of the individual copolymers and the subsequent behavior of the entire self-assembled vesicles that they form.

Temperature-indifferent polymersomes assembled from $\mathrm{PMOXA}_{14}-\mathrm{PDMS}_{65}-\mathrm{PMOXA}_{14}$ were also studied with SANS. In Figure 4(d), the scattering curves and model fits of the $\mathrm{PMOXA}_{14}-\mathrm{PDMS}_{65}-\mathrm{PMOXA}_{14}$ polymersomes at $25{ }^{\circ} \mathrm{C}$ and $55^{\circ} \mathrm{C}$, vertically offset for clarity, show the complete absence of temperature-induced morphological change since the shapes of the two scattering curves are identical. Calculations from the model fits at both temperatures gave a size of $162 \mathrm{~nm}$ with a $13 \mathrm{~nm}$ shell thickness. The size of polymersomes at low and high temperature are in good agreement with the DLS data, while the lack of thickness change is attributed to PMOXA retaining its hydrophilic character at high temperature. The shell thickness of polymersomes formed from $\mathrm{PMOXA}_{17}-\mathrm{PDMS}_{67}$ PMOXA $_{17}$ copolymers was previously shown to be $11.7 \mathrm{~nm}$ via cryo-TEM,${ }^{69}$ which is in agreement with the thickness we found for the PMOXA $14-\mathrm{PDMS}_{65}-\mathrm{PMOXA}_{14}$ polymersomes in our SANS studies. The slight apparent increase in shell thickness in our calculation is a result of including the solvated hydrophilic corona of the polymersome shell as a portion of the total SANS contrast, which cannot be observed by electron microscopy.

\section{Effect of Hydrophilic Ratio $(f)$ and PVCL Chain Length on Temperature-Induced Size and Thickness Changes in PVCL-PDMS-PVCL Polymersomes}

Figure 5(a) and (b) shows the SANS scattering curves of $\mathrm{PVCL}_{15}-\mathrm{PDMS}_{65}-\mathrm{PVCL}_{15}$ and $\mathrm{PVCL}_{21}-\mathrm{PDMS}_{65}-\mathrm{PVCL}_{21}$ polymersomes, fitted with the same core-shell model as described above. As the solution temperature was increased from 25 to $55^{\circ} \mathrm{C}$, the diameter of the $\mathrm{PVCL}_{15}-\mathrm{PDMS}_{65}-\mathrm{PVCL}_{15}$ and $\mathrm{PVCL}_{21}-\mathrm{PDMS}_{65}-\mathrm{PVCL}_{21}$ polymersomes decreased from 206 to $178 \mathrm{~nm}$ and from 183 to $167 \mathrm{~nm}$, respectively. The 13 and 9\% change in vesicle diameter extracted from the model fits is in good agreement with the DLS data shown in Table 1. The shell thickness of the $\mathrm{PVCL}_{15}-\mathrm{PDMS}_{65}-\mathrm{PVCL}_{15}$ and $\mathrm{PVCL}_{21}-\mathrm{PDMS}_{65}$ $\mathrm{PVCL}_{21}$ polymersomes decreased from 16 to $13 \mathrm{~nm}$ and from 19.1 to $13.6 \mathrm{~nm}$, respectively, representing a decrease of 19 and $29 \%$. 
In comparing the shell thickness of the $\mathrm{PVCL}_{n}-\mathrm{PDMS}_{65}-\mathrm{PVCL}_{\mathrm{n}}$ polymersomes with PVCL $\mathrm{n}=10,15$, and 21 (Table 2), the expected trend of increasing shell thickness with increasing PVCL block length can be seen. This observation is supported by a recent study in which the shell thickness of PMOXA-PDMS-PMOXA polymersomes increased from 6 to $13.4 \mathrm{~nm}$ when the molecular weight of the copolymers increased from 2000 to $7000 \mathrm{Da}$, which was measured by cryo-TEM. ${ }^{69}$ Similarly, it has also been shown that polymersomes assembled from block copolymers of poly(ethylene oxide)-poly(butadiene) had shell thickness of 9.6 and $14.8 \mathrm{~nm}$ when the copolymer molecular weights were 3600 and $10400 \mathrm{Da}$, respectively. ${ }^{70}$ Interestingly, it has also been demonstrated that the hydrophobic region of polymersomes assembled from higher hydrophilic ratios showed a more stretched chain architecture, while lower hydrophilic ratios exhibited interdigitation of the hydrophilic blocks into the hydrophobic region. ${ }^{39}$

An important trend revealed in this study is that the polymersomes with higher $f$ ratios had more significant temperature induced shell shrinkage. As the PVCL chains attached to both inner and outer faces of the membrane dehydrate above the LCST, the longer chains exhibit a greater magnitude of reduction in size compared to shorter PVCL chains, and the thickness of the polymersome shell followed accordingly. Our results indicate the interesting prospect of polymersomes with tunable membrane thickness (up to 1.4 fold) controlled by temperature.

To further explore the effect of $f$ and PVCL chain length on temperature responsive polymersomes, we also characterized polymersomes assembled from $\mathrm{PVCL}_{5} \mathrm{PDMS}_{30^{-}}$ $\mathrm{PVCL}_{5}(f=0.33)$ and $\mathrm{PVCL}_{7}-\mathrm{PDMS}_{47}-\mathrm{PVCL}_{7}(f=0.36)$. We characterized the thickness of the vesicle membrane assembled from $\mathrm{PVCL}_{5}-\mathrm{PDMS}_{30}-\mathrm{PVCL}_{5}$ by cryo-TEM (Figure $\mathrm{S} 4$ (b)), finding the thickness to be $9.3 \pm 1.5 \mathrm{~nm}$. The shell thickness as measured from cryoTEM is in excellent agreement with that extracted from the scattering curves of the same polymer at room temperature $(8.5 \mathrm{~nm})$. Figure $5(\mathrm{c})$ and $(\mathrm{d})$ shows the SANS scattering curves for the polymersomes formed from $\mathrm{PVCL}_{7}-\mathrm{PDMS}_{47}-\mathrm{PVCL}_{7}$ and $\mathrm{PVCL}_{5}-\mathrm{PDMS}_{30^{-}}$ $\mathrm{PVCL}_{5}$ at 25 and $55^{\circ} \mathrm{C}$. The extracted fit parameters show that while the $\mathrm{PVCL}_{7}-\mathrm{PDMS}_{47^{-}}$ $\mathrm{PVCL}_{7}$ polymersomes exhibited a $15 \%$ shrinkage in membrane thickness, the membrane thickness of $\mathrm{PVCL}_{5}-\mathrm{PDMS}_{30}-\mathrm{PVCL}_{5}$ only decreased by $6 \%$. Interestingly, although it was expected that copolymers with similar $f$ ratios would exhibit similar extents of temperature responsiveness, the polymersomes formed from copolymers with similar $f$ ratios in our study $\left(\mathrm{PVCL}_{5}-\mathrm{PDMS}_{30}-\mathrm{PVCL}_{5}(f=0.33), \mathrm{PVCL}_{7}-\mathrm{PDMS}_{47}-\mathrm{PVCL}_{7}(f=0.36)\right.$, and $\mathrm{PVCL}_{10^{-}}$ $\left.\mathrm{PDMS}_{65}-\mathrm{PVCL}_{10}(f=0.36)\right)$ exhibited similar size reductions at elevated temperature $(21 \%$, $18 \%$, and $18 \%$, respectively) but the thickness shrinkage decreased with decreasing chain length. From the data above, we concluded that the longer PVCL blocks in PVCL $\mathrm{P}^{-}$ $\mathrm{PDMS}_{65}-\mathrm{PVCL}_{21}$ and $\mathrm{PVCL}_{10}-\mathrm{PDMS}_{65}-\mathrm{PVCL}_{10}$ experienced a greater collapse at $55^{\circ} \mathrm{C}$ than those in the $\mathrm{PVCL}_{7}-\mathrm{PDMS}_{47}-\mathrm{PVCL}_{7}$ and $\mathrm{PVCL}_{5}-\mathrm{PDMS}_{30}-\mathrm{PVCL}_{5}$ polymersomes. However, the reduction of shell thickness from $\mathrm{PVCL}_{7}-\mathrm{PDMS}_{47}-\mathrm{PVCL}_{7}$ and $\mathrm{PVCL}_{5}$ $\mathrm{PDMS}_{30}-\mathrm{PVCL}_{5}$ polymersomes may follow a different physical mechanism compared to $\mathrm{PVCL}_{10}-\mathrm{PDMS}_{65}-\mathrm{PVCL}_{10}$ polymersomes. Although we have discussed the effect of $f$ value based on the same length of hydrophobic block $\left(\mathrm{PDMS}_{65}\right)$, the effect of the PVCL block length was not easily explained from the SANS data alone. For this, we utilized MD simulations to gain insight into the potential mechanism. 


\section{Simulations of Temperature-Induced Compaction of Single PVCL Blocks}

To elucidate the molecular-level origin of the length dependence of the response of the PVCL block to temperature change, we built all-atom models of $\mathrm{PVCL}_{5}, \mathrm{PVCL}_{7}$ and $\mathrm{PVCL}_{10}$ polymers in an aqueous environment. Figure 6(a) illustrates the initial state of one such system (see MD methods for a detailed description of the modeling procedures). Following energy minimization, the models were simulated using the all-atom MD method at $275,295,315$ and $328 \mathrm{~K}$ for approximately $300 \mathrm{~ns}$. Four independent simulations were performed for each polymer length and temperature condition. Figure 6(b) plots the average end-to-end distance of the PVCL polymers as a function of temperature (see Figures S5-7 for individual end-to-end traces). The length of the $\mathrm{PVCL}_{10}$ polymer was found to decrease with temperature, reducing by $25 \%$ at $328 \mathrm{~K}$ with respect to the $275 \mathrm{~K}$ length. Considerably less pronounced reduction of the polymer length was observed for $\mathrm{PVCL}_{7}$ and $\mathrm{PVCL}_{5}$ polymers: 13 and $5 \%$, respectively. Thus, we found longer polymers to contract considerably more than the shorter ones, even when the contraction ratio is normalized by the number of monomers in each polymer. Thus, we can predict that the volume transition for $\mathrm{PVCL}_{15}$ and $\mathrm{PVCL}_{21}$ blocks will occur to a greater degree than for $\mathrm{PVCL}_{10}$, which is consistent with our experimental observations where longer copolymers were found to exhibit a larger degree of shell shrinkage above the LCST (328 K).

Analysis of the simulation trajectories provided a molecular-level explanation for the observed dependence. Figure 6(c) illustrates the conformations of $\mathrm{PVCL}_{5}$ and $\mathrm{PVCL}_{10}$ at $275 \mathrm{~K}$ and $328 \mathrm{~K}$. The conformation of $\mathrm{PVCL}_{5}$ shows little temperature dependence, whereas $\mathrm{PVCL}_{10}$ appears to undergo an extended chain-to-globule-like transition when temperature changed from 275 to $328 \mathrm{~K}$. The animations of the MD trajectories ( Movies S1-4 and Figure S8), show that $\mathrm{PVCL}_{n}$ is a relatively rigid polymer due to the large hindrance from the caprolactam ring structures. Hence, the probability for non-neighboring monomers to interact with one another depends on the distance between the units on the polymer backbone. As the association of the hydrophobic portion from the PVCL polymer is the driving force of the polymer phase transition, ${ }^{71}$ the shorter PVCL blocks lack the flexibility to overcome the steric hindrance from the caprolactam rings, which explains the observed dependence of temperature-induced size change on polymer length.

The collapse of the PVCL region of our triblock copolymer is directly related to the shrinkage of the vesicle membrane at elevated temperature. Figure 6(b) illustrates that the reduction of end-to-end distance of $\mathrm{PVCL}_{10}$ is around $0.4 \mathrm{~nm}$, which means the contribution of shell shrinkage of $\mathrm{PVCL}_{10}-\mathrm{PDMS}_{65}-\mathrm{PVCL}_{10}$ vesicles from the PVCL blocks (the sum of inner and outer blocks) should approach $0.8 \mathrm{~nm}$ at $328 \mathrm{~K}$. However, the total reduction of shell thickness according to our SANS analysis was $2.4 \mathrm{~nm}$ (Table 2). This may be due to an alteration of PDMS chain conformation by the change of effective hydrophilicity of the PVCL block at $328 \mathrm{~K}$. Because PVCL blocks are located in both inner and outer regions of the vesicle membrane, the following two driving forces may affect the conformation of the PDMS chains: hydrophobic association between different PDMS segments and stretching of the PDMS segments due to the dynamic PVCL chains. The hydrophobic association between PDMS chains does not exhibit strong temperature dependence, ${ }^{72}$ where the tug-ofwar pulling from the two PVCL blocks can be substantial because of the pronounced 
reduction of hydrophilicity at high temperature. At ambient temperature, the PDMS region adopts a more extended conformation due to the outward pull from the solvated PVCL blocks extending into the solvent, whereas at elevated temperature the PVCL blocks expel water, pushing inward toward the PDMS membrane and contributing to the reduction of shell thickness of the PDMS. For vesicles assembled from the shorter copolymers ( $\mathrm{PVCL}_{7}$ $\mathrm{PDMS}_{47}-\mathrm{PVCL}_{7}$ and $\mathrm{PVCL}_{5}-\mathrm{PDMS}_{30}-\mathrm{PVCL}_{5}$ ) the $\mathrm{PVCL}$ regions contribute less to the shell shrinkage ( $0.3 \mathrm{~nm}$ and $0.1 \mathrm{~nm}$, respectively) because of the less pronounced dependence of the $\mathrm{PVCL}_{7}$ and $\mathrm{PVCL}_{5}$ single block length on temperature. Thus, the combination of vesicle shell characterization experiments and single PVCL block simulations allows us to delineate the behaviors of the hydrophilic and hydrophobic blocks through the LCST transition and provides guidance for choosing the $f$ value and the hydrophilic chain length in future design of temperature responsive polymersomes.

\section{Temperature Responsive Structural Changes and Release Mechanism}

We have shown recently that polymersomes formed from $\mathrm{PVCL}_{\mathrm{n}}-\mathrm{PDMS}_{65}-\mathrm{PVCL}_{\mathrm{n}}$ with PVCL $\mathrm{n}=10,15$, and 19 exhibited temperature-responsive release of DOX. ${ }^{31}$ In that work, the change in vesicle architecture allowed the small molecule to diffuse out of the polymersomes. However, while the volume of polymersomes rapidly decreased to $53 \%$ of the original size, the loaded drug was continuously released over 24 hours. This indicated that the mechanism of drug release from these temperature-responsive polymersomes is more complicated than simply escaping in concert with the volume transition. While polymersome shrinkage is likely an important step in the release process, the sustained release suggests a pathway is opened to allow the relatively hydrophilic small molecules to permeate through the hydrophobic shell. In the context of our previous drug release data and the current SANS study on the polymersome shell, we propose a possible mechanism of drug release through the polymersome membrane.

Based on the pronounced change in shell thickness for the $\mathrm{PVCL}_{21}-\mathrm{PDMS}_{65}-\mathrm{PVCL}_{21}$ polymersomes above the LCST that we discovered here, it could be expected that similar or even enhanced release of DOX at high temperature would have been observed in our previous study for copolymers with higher $f$ ratios if membrane thickness alone was solely responsible. However, our previous work showed that the $\mathrm{PVCL}_{10}-\mathrm{PDMS}_{65}-\mathrm{PVCL}_{10}$ polymersomes released $85 \%$ of the loaded DOX at $42{ }^{\circ} \mathrm{C}$ while the $\mathrm{PVCL}_{15}-\mathrm{PDMS}_{65^{-}}$ $\mathrm{PVCL}_{15}$ and $\mathrm{PVCL}_{19}-\mathrm{PDMS}_{65}-\mathrm{PVCL}_{19}$ polymersomes exhibited less drug release (45\% and $20 \%$, respectively). By analyzing the diffusion coefficient of the drug at different temperatures, we found that the diffusion coefficient of the loaded DOX increased drastically around $42{ }^{\circ} \mathrm{C}$. But in terms of structural change, the $\mathrm{PVCL}_{10}-\mathrm{PDMS}_{65}-\mathrm{PVCL}_{10}$ polymersomes exhibited a gradual size decrease from 25 to $55^{\circ} \mathrm{C}$. We can thus conclude that the drug release from $\mathrm{PVCL}_{10}-\mathrm{PDMS}_{65}-\mathrm{PVCL}_{10}$ polymersomes is not solely related to the size reduction. Importantly, the hydrophilic/hydrophobic transition of the PVCL corona on the polymersome membrane affects the hydrophobic association of the PDMS region within the membrane, which directly influences the rigidity and fluidity of the PDMS responsible for forming the hydrophobic membrane. ${ }^{69}$ Tang et al illustrated that the thermoresponsive hydrophilic block on unilamellar vesicles can intercalate into the hydrophobic region above its LCST, and that his process is reversible. ${ }^{73}$ In our case, the 
PVCL blocks exhibited increasing hydrophobicity and steadily collapsed during the heating ramp. During this temperature transition, they may intermingle with the inner PDMS portion of the polymersome membrane as they further repel water molecules and the fluidity of PDMS increases. ${ }^{74}$ Above $42{ }^{\circ} \mathrm{C}$, the integrity of the vesicles was preserved but the homogeneity of the hydrophobic region was affected by the intermixing of the PVCL. In support of this intermixing behavior, we observed an increase in the SLD of the shell at $55^{\circ} \mathrm{C}$ in our SANS studies of the PVCL-PDMS-PVCL polymersomes. As the SLD of PDMS is much lower than that of $\mathrm{D}_{2} \mathrm{O}$ and hydrated PVCL, an increase in the overall shell SLD could indicate intermixing of the minimally hydrated PVCL and ingress of small amounts of $\mathrm{D}_{2} \mathrm{O}$ alongside it. This could indicate a possible mechanism for sustained release of DOX from the $\mathrm{PVCL}_{10}-\mathrm{PDMS}_{65}-\mathrm{PVCL}_{10}$ polymersomes, as the intermixing of PVCL within the hydrophobic PDMS region of the membrane could create dynamic channels of lower hydrophobicity for passage of hydrophilic payload molecules. That the mechanism of temperature-controlled release in our polymersomes likely relies on dynamic porosity of the membrane in the absence of changes to their structural integrity is an interesting characteristic, especially when contrasted with non-temperature-responsive carriers like the $\mathrm{PMOXA}_{14}-\mathrm{PDMS}_{65}-\mathrm{PMOXA}_{14}$ polymersomes used for comparison. Each of the characterization methods utilized in our study showed no change in size or shell thickness for the PMOXA $_{14}-\mathrm{PDMS}_{65}-\mathrm{PMOXA}_{14}$ polymersomes at elevated temperatures, which is in agreement with reports on their characteristic stability. ${ }^{75,76}$ In those studies, the release of cargo from the polymersomes was more likely to occur as a result of membrane degradation. This distinction between cargo release mechanisms may open branches in the path to specialized controlled-release vehicles in which the structural integrity of the carrier pre- and post-release can also be a considering factor in their application.

Although the polymersomes formed from $\mathrm{PVCL}_{10}-\mathrm{PDMS}_{65}-\mathrm{PVCL}_{10}$ exhibited favorable drug release capability, the polymersomes made by the copolymers with higher PVCL content performed increasingly poorly. The most likely explanation is that for longer PVCL blocks, the interchain entanglement of the PVCL blocks on the inner and outer surface of the membrane seals the shell with a steric barrier in their collapsed state (Figure 7). In this regard, due to their length, the collapsed shell actually became much denser, and the loaded drug is less able to pass through. Additionally, the increased chain entanglement for the longer PVCL chains likely prohibits substantial intermixing of the chains within the inner PDMS portion of the membrane. This lower degree of intermixing is supported by the SANS data at elevated temperature for the polymersomes with longer PVCL chains. While the $\mathrm{PVCL}_{10}-\mathrm{PDMS}_{65}-\mathrm{PVCL}_{10}$ polymersomes experienced a $25 \%$ increase in shell SLD (Table S2) at $55^{\circ} \mathrm{C}$, the $\mathrm{PVCL}_{15}-\mathrm{PDMS}_{65}-\mathrm{PVCL}_{15}$ and $\mathrm{PVCL}_{21}-\mathrm{PDMS}_{65}-\mathrm{PVCL}_{21}$-based polymersomes exhibited only 13 and $4 \%$ increases in their shell SLDs, respectively, indicating a drastically lowered intermixing of PVCL and/or $\mathrm{D}_{2} \mathrm{O}$.

As described above, the extent of the effects of the hydrophilic-to-hydrophobic transition of PVCL in the polymersome shell caused the difference in drug release behavior and was dependent on the length of the PVCL chains. The mechanism of the polymersome size reduction can be also expanded to include the differences in the hydrophilic-to-hydrophobic transitions between these polymers. We have shown that a higher $f$ for a copolymer is an excellent predictor for vesicle size decrease at elevated temperature, allowing the PVCL 
chains to more densely pack at the surface in the process. We also described above how the intermixing of PVCL into the PDMS portion of the shell served to increase its permeability and neutron SLD. From our analysis, the shell permeability is likely a coordinated relationship between shorter PVCL chains that can intercalate into the PDMS region of the shell and a thinning PDMS membrane that create dynamic pathways for passage of hydrophilic payload molecules through the otherwise impenetrable hydrophobic barrier. The lower $f$ polymersomes exhibited increased changes in their size, which could be influenced by the increased instability and fluidity, especially at elevated temperatures, of a membrane with PDMS and PVCL intermixed. In the case of the highly intermixed membranes, less hydrophilic interactions between the PVCL and solvent could likely serve to force the shells into smaller conformations where their slightly increased density may help stabilize their morphology. For the polymersomes with higher $f$ values, the more exaggerated increase in shell density and hydrophobic association coming from the collapse of PVCL with high chain entanglement may stabilize the shell against a major size change. In addition, the longer PVCL chains are unable to fully intermix into the PDMS, leaving more PVCL near the solvation boundary to help stabilize the polymersome shell in the aqueous solvent. The data provides a framework for optimizing triggered release, loading capacity, and thermoresponse of polymersomes by controlling the hydrophilic-to-hydrophobic ratio, $f$, and total chain length. Our results and observations reinforce the importance of the hydrophilic ratio on the ability of these temperature-responsive polymersomes to exhibit dynamic size and shell thickness changes, and the exploration of the underlying mechanisms helps us precisely design the next generation of advanced drug carriers.

\section{Conclusions}

We reported on in situ characterization of temperature-responsive $\mathrm{PVCL}_{\mathrm{n}}-\mathrm{PDMS}_{\mathrm{m}}-\mathrm{PVCL}_{\mathrm{n}}$ and non-temperature responsive $\mathrm{PMOXA}_{14}-\mathrm{PDMS}_{65}-\mathrm{PMOXA}_{14}$ polymersomes at 25 and $55^{\circ} \mathrm{C}$ via DLS, TEM, and SANS. This work explored the relationship between the $f$ value of the copolymer and physical changes in its resulting $\mathrm{PVCL}_{\mathrm{n}}-\mathrm{PDMS}_{\mathrm{m}}-\mathrm{PVCL}_{\mathrm{n}}$ polymersome during temperature changes through the LCST range. These polymersomes collapse into smaller vesicles with thinner shells above the LCST, but the extent of each transformation is highly dependent on the hydrophilic-to-hydrophobic ratio $f$. PMOXA $_{14}-\mathrm{PDMS}_{65}-\mathrm{PMOXA}_{14}$ polymersomes used as controls retained their original structure at all temperature ranges due to the lack of temperature-responsive polymer constituents. We found that the temperatureinduced polymersome diameter reduction was influenced heavily by the extent of PVCL chain collapse and possible subsequent intermixing into the PDMS layer. As PVCL chain length increases, the associated polymersomes exhibit smaller sizes and thicker shells at room temperature, but increased shell thickness change and decreased size change at higher temperature. MD simulation determined $\mathrm{PVCL}_{5}$ chains to be too short to exhibit chain collapse at $55{ }^{\circ} \mathrm{C}$ due to the rigid backbone, in accord with the small shell thickness change observed in the corresponding polymersomes. We believe the exploration of these mechanisms and the analysis of our results clarifies the understanding of temperatureresponsive polymersomes for stimulus responsive cargo release. 


\title{
Supplementary Material
}

Refer to Web version on PubMed Central for supplementary material.

\section{ACKNOWLEDGMENT}

\begin{abstract}
This work was supported US Army Corps of Engineers CERL, W9132T-17-2-0008 and NSF-DMR 1608728 (EK), and the National Institute of Health P41-GM104601 (AlAk). UAB High Resolution Imaging Facility (TEM) is also acknowledged. We thank Cynthia Rodenburg for carrying out the cryo-EM work in the UAB Cryo-EM facility (UAB Department of Microbiology). Neutron scattering research conducted at the Bio-SANS instrument, a DOE Office of Science, Office of Biological and Environmental Research resource, used resources at the High Flux Isotope Reactor, a DOE Office of Science, Scientific User Facility operated by the Oak Ridge National Laboratory. The supercomputer time provided through XSEDE Allocation Grant MCA05S028 and the Blue Waters petascale supercomputer system (UIUC) is acknowledged.
\end{abstract}

\section{References}

(1). Masood F Polymeric Nanoparticles for Targeted Drug Delivery System for Cancer Therapy. Mater. Sci. Eng. C 2016, 60, 569-578.

(2). Zhang R; Fan Q; Yang M; Cheng K; Lu X; Zhang L; Huang W; Cheng Z Engineering Melanin Nanoparticles as an Efficient Drug-Delivery System for Imaging-Guided Chemotherapy. Adv. Mater. 2015, 27, 5063-5069. [PubMed: 26222210]

(3). Bharti C; Nagaich U; Pal AK; Gulati N Mesoporous Silica Nanoparticles in Target Drug Delivery System: A Review. Int. J. Pharma. Investig. 2015, 5, 124.

(4). Battaglia G; Ryan AJ Bilayers and Interdigitation in Block Copolymer Vesicles. J. Am. Chem. Soc. 2005, 127, 8757-8764. [PubMed: 15954782]

(5). Discher BM; Won Y-Y; Ege DS; Lee JC; Bates FS; Discher DE; Hammer DA Polymersomes: Tough Vesicles Made from Diblock Copolymers. Science 1999, 284, 1143-1146. [PubMed: 10325219]

(6). Meng F; Hiemstra C; Engbers GH; Feijen J Biodegradable Polymersomes. Macromolecules 2003, 36, 3004-3006.

(7). Soussan E; Cassel S; Blanzat M; Rico-Lattes I Drug Delivery by Soft Matter: Matrix and Vesicular Carriers. Angew. Chem. Int. Ed. 2009, 48, 274-288.

(8). Ahmed F; Pakunlu RI; Brannan A; Bates F; Minko T; Discher DE Biodegradable Polymersomes Loaded with Both Paclitaxel and Doxorubicin Permeate and Shrink Tumors, Inducing Apoptosis in Proportion to Accumulated Drug. J. Control. Release 2006, 116, 150-158. [PubMed: 16942814]

(9). Mai Y; Eisenberg A Self-Assembly of Block Copolymers. Chem. Soc. Rev. 2012, 41, 5969-5985. [PubMed: 22776960]

(10). Wang Y; Sukhishvili SA All-Aqueous Nanoprecipitation: Spontaneous Formation of HydrogenBonded Nanoparticles and Nanocapsules Mediated by Phase Separation of Poly(NIsopropylacrylamide). Macromol. Rapid Commun. 2017, 38, 1700242.

(11). Wang Y; Sukhishvili SA Hydrogen-Bonded Polymer Complexes and Nanocages of Weak Polyacids Templated by a Pluronic @ Block Copolymer. Soft Matter 2016, 12, 8744-8754. [PubMed: 27722711]

(12). Ogunsola OA; Kraeling ME; Zhong S; Pochan DJ; Bronaugh RL; Raghavan SR Structural Analysis of "Flexible" Liposome Formulations: New Insights into the Skin-Penetrating Ability of Soft Nanostructures. Soft Matter 2012, 8, 10226-10232.

(13). Kim HR; You DG; Park S-J; Choi K-S; Um W; Kim J-H; Park JH; Kim Y.-s. MRI monitoring of tumor-selective anticancer drug delivery with stable thermosensitive liposomes triggered by highintensity focused ultrasound. Mol. Pharm. 2016, 13, 1528-1539. [PubMed: 26998616]

(14). Sercombe L; Veerati T; Moheimani F; Wu SY; Sood AK; Hua S Advances and challenges of liposome assisted drug delivery. Front Pharmacol 2015, 6, 286. [PubMed: 26648870]

(15). Onaca O; Enea R; Hughes DW; Meier W Stimuli-Responsive Polymersomes as Nanocarriers for Drug and Gene Delivery. Macromol. Biosci. 2009, 9, 129-139. [PubMed: 19107717] 
(16). Li M-H; Keller P Stimuli-Responsive Polymer Vesicles. Soft Matter 2009, 5, 927-937.

(17). Kim KT; Cornelissen JJ; Nolte RJ; Van Hest JC A Polymersome Nanoreactor with Controllable Permeability Induced by Stimuli-Responsive Block Copolymers. Adv. Mater. 2009, 21, $2787-$ 2791.

(18). Ghoroghchian PP; Li G; Levine DH; Davis KP; Bates FS; Hammer DA; Therien MJ Bioresorbable Vesicles Formed Through Spontaneous Self-Assembly of Amphiphilic Poly(ethylene oxide)-block-Polycaprolactone. Macromolecules 2006, 39, 1673-1675. [PubMed: 20975926]

(19). Liao J; Wang C; Wang Y; Luo F; Qian Z Recent Advances in Formation, Properties, and Applications of Polymersomes. Curr. Pharm. Des. 2012, 18, 3432-3441. [PubMed: 22632981]

(20). Zhu Z; Gao N; Wang H; Sukhishvili SA Temperature-Triggered On-Demand Drug Release Enabled by Hydrogen-Bonded Multilayers of Block Copolymer Micelles. J. Control. Release 2013, 171, 73-80. [PubMed: 23831052]

(21). Gebhardt KE; Ahn S; Venkatachalam G; Savin DA Role of Secondary Structure Changes on the Morphology of Polypeptide-Based Block Copolymer Vesicles. J. Colloid Interface Sci. 2008, 317, 70-76 [PubMed: 17936292]

(22). Fang JS; Gillies RD; Gatenby RA Adaptation to Hypoxia and Acidosis in Carcinogenesis and Tumor Progression. Semin. Cancer Biol. 2008, 18, 330-337. [PubMed: 18455429]

(23). Vácha R; Siu SW; Petrov M; ckmann RA; Barucha-Kraszewska J; Jurkiewicz P; Hof M; Berkowitz ML; Jungwirth P Effects of Alkali Cations and Halide Anions on the DOPC Lipid Membrane. J. Phys. Chem. A 2009, 113, 7235-7243. [PubMed: 19290591]

(24). Davis JH; Clair JJ; Juhasz J Phase Equilibria in DOPC/DPPC-d62/Cholesterol Mixtures. Biophys. J. 2009, 96, 521-539. [PubMed: 19167302]

(25). Vemuri S; Rhodes C Preparation and Characterization of Liposomes as Therapeutic Delivery Systems: A Review. Pharm. Acta Helv. 1995, 70, 95-111. [PubMed: 7651973]

(26). Agut W; Brûlet A; Schatz C; Taton D; Lecommandoux S pH and Temperature Responsive Polymeric Micelles and Polymersomes by Self-Assembly of Poly [2-(dimethylamino) ethyl methacrylate]-b-poly (glutamic acid) Double Hydrophilic Block Copolymers. Langmuir 2010, 26, 10546-10554. [PubMed: 20491497]

(27). Li Y; Lokitz BS; McCormick CL Thermally Responsive Vesicles and Their Structural "Locking" Through Polyelectrolyte Complex Formation. Angew. Chem. Int. Ed. 2006, 45, 5792-5795.

(28). Liang X; Liu F; Kozlovskaya V; Palchak Z; Kharlampieva E Thermoresponsive Micelles from Double LCST-poly(3-methyl-N-vinylcaprolactam) Block Copolymers for Cancer Therapy. ACS Macro Lett. 2015, 4, 308-311.

(29). Kita-Tokarczyk K; Grumelard J; Haefele T; Meier W Block Copolymer Vesicles - Using Concepts from Polymer Chemistry to Mimic Biomembranes. Polymer 2005, 4, 3540-3563.

(30). Marguet M; Sandre O; Lecommandoux S Polymersomes in "Gelly" Polymersomes: Toward Structural Cell Mimicry. Langmuir 2012, 28, 2035-2043. [PubMed: 22149733]

(31). Liu F; Kozlovskaya V; Medipelli S; Xue B; Ahmad F; Saeed M; Cropek D; Kharlampieva E Temperature-Sensitive Polymersomes for Controlled Delivery of Anticancer Drugs. Chem. Mater. 2015, 27, 7945-7956.

(32). Wei H; Cheng S-X; Zhang X-Z; Zhuo R-X Thermo-Sensitive Polymeric Micelles Based on Poly(N-isopropylacrylamide) as Drug Carriers. Prog. Polym. Sci. 2009, 34, 893-910.

(33). Qin S; Geng Y; Discher DE; Yang S Temperature-Controlled Assembly and Release from Polymer Vesicles of Poly (ethylene oxide)-block-poly (N-isopropylacrylamide). Adv. Mater. 2006, 18, 2905-2909.

(34). Smith AE; Xu X; Savin DA; McCormick CL Reversible Gold "Locked" Synthetic Vesicles Derived from Stimuli-Responsive Diblock Copolymers. Polym. Chem. 2010, 1, 628-630.

(35). Sauer M; Meier W Responsive Nanocapsules. Chem. Commun. 2001, 1, 55-56.

(36). Sauer M; Haefele T; Graff A; Nardin C; Meier W Ion-Carrier Controlled Precipitation of Calcium Phosphate in Giant ABA Triblock Copolymer Vesicles. Chem. Commun. 2001, 23, 2452-2453. 
(37). Robinson N Molecular Size and Shape: A Review of the Light-Scattering Method Applied to Some Important Biological and other Macromolecules. Part I. J. Pharm. Pharmacol. 1960, 12, 129-149. [PubMed: 14437979]

(38). Kim KT; Meeuwissen SA; Nolte RJ; Van Hest JC Smart Nanocontainers and Nanoreactors. Nanoscale 2010, 2, 844-858. [PubMed: 20648280]

(39). Discher DE; Eisenberg A Polymer Vesicles. Science 2002, 297, 967-973. [PubMed: 12169723]

(40). Zhang L; Eisenberg A Multiple Morphologies of "Crew-Cut" Aggregates of Polystyrene-b-poly (acrylic acid) Block Copolymers. Science 1995, 268, 1728-1731. [PubMed: 17834990]

(41). Bermudez H; Brannan AK; Hammer DA; Bates FS; Discher DE Molecular Weight Dependence of Polymersome Membrane Structure, Elasticity, and Stability. Macromolecules 2010, 35 (21), 8203-8208.

(42). Alford A; Kozlovskaya V; Kharlampieva E, Small Angle Scattering for Pharmaceutical Applications: From Drugs to Drug Delivery Systems In Biological Small Angle Scattering: Techniques, Strategies and Tips, Springer, Singapore, 2017; 239-262.

(43). Kučerka N; Kiselev MA; Balgavý P Determination of Bilayer Thickness and Lipid Surface Area in Unilamellar Dimyristoylphosphatidylcholine Vesicles from Small-Angle Neutron Scattering Curves: A Comparison of Evaluation Methods. Eur. Biophys. J. 2004, 33, 328-334. [PubMed: 12955364]

(44). Hocine S; Cui D; Rager M-N; Di Cicco A; Liu J-M; Wdzieczak-Bakala J; Brûlet A; Li M-H Polymersomes with PEG Corona: Structural Changes and Controlled Release Induced by Temperature Variation. Langmuir 2013, 29, 1356-1369. [PubMed: 23293844]

(45). Salva R; Le Meins J-F; Sandre O; Brûlet A; Schmutz M; Guenoun P; Lecommandoux S Polymersome Shape Transformation at the Nanoscale. ACS Nano 2013, 7, 9298-9311. [PubMed: 24047230]

(46). Zhang Y; Zhao W; Yang J; Hammouda B; Yang J; Cheng G SANS Study on Self-Assembled Structures of Glucose-Responsive Phenylboronate Ester-Containing Diblock Copolymer. Eur. Polym. J. 2016, 83, 173-180.

(47). Kline SR Reduction and Analysis of SANS and USANS Data using IGOR Pro. J. Appl. Crystallogr. 2006, 39, 895-900.

(48). Phillips JC; Braun R; Wang W; Gumbart J; Tajkhorshid E; Villa E; Chipot C; Skeel RD; Kale L; Schulten K Scalable Molecular Dynamics with Namd. J. Comput. Chem. 2005, 26, 1781-1802. [PubMed: 16222654]

(49). MacKerell AD; Bashford D; Bellott MLDR; Dunbrack RL Jr; Evanseck JD; Field MJ; Fischer S; Gao J; Guo H; Ha S; Joseph-McCarthy D All-Atom Empirical Potential for Molecular Modeling and Dynamics Studies of Proteins. J. Phys. Chem. B 1998, 102, 3586-3616. [PubMed: 24889800]

(50). Yoo J; Aksimentiev A New tricks for Old Dogs: Improving the Accuracy of Biomolecular Force Fields by Pair-Specific Corrections to Non-Bonded Interactions. Phys. Chem. Chem. Phys. 2018, 20, 8432-8449. [PubMed: 29547221]

(51). Andersen HC Rattle: A "Velocity" Version of the Shake Algorithm for Molecular Dynamics Calculations. J. Comput. Phys. 1983, 52, 24-34.

(52). Miyamoto S; Kollman PA Settle: An Analytical Version of the SHAKE and RATTLE Algorithm for Rigid Water Models. J. Comput. Chem. 1992, 13, 952-962.

(53). Darden T; York D; Pedersen L Particle Mesh Ewald: An N· $\log (N)$ Method for Ewald Sums in Large Systems. J. Chem. Phys. 1993, 98, 10089-10092.

(54). Martyna GJ; Tobias DJ; Klein ML Constant Pressure Molecular Dynamics Algorithms. J. Chem. Phys. 1994, 101, 4177-4189.

(55). Brünger AT X-PLOR: Version 3.1: A System for X-ray Crystallography and NMR. Yale University Press, New Haven, 1992.

(56). Csizmadia P In MarvinSketch and MarvinView: Molecule Applets for the World Wide Web, Proceedings of ECSOC-3, The Third International Electronic Conference on Synthetic Organic Chemistry, 1999; 1999; 367-369. 
(57). Hanwell MD; Curtis DE; Lonie DC; Vandermeersch T; Zurek E; Hutchison GR Avogadro: An Advanced Semantic Chemical Editor, Visualization, and Analysis Platform. J. Cheminformatics 2012, 4, 17.

(58). Nardin C; Thoeni S; Widmer J; Winterhalter M; Meier W Nanoreactors Based on (Polymerized) ABA-Triblock Copolymer Vesicles. Chem. Commun.. 2000, 1433-1434.

(59). Liang X; Kozlovskaya V; Cox CP; Wang Y; Saeed M; Kharlampieva E Synthesis and selfassembly of Thermosensitive Double-hydrophilic Poly(N-vinylcaprolactam)-b-poly(N-vinyl-2pyrrolidone) Diblock Copolymers. J. Polym. Sci. A: Polym. Chem. 2014, 52, 2725-2737.

(60). Nemethy G; Scheraga HA Structure of Water and Hydrophobic Bonding in Proteins. IV. The Thermodynamic Properties of Liquid Deuterium Oxide. J. Chem. Phys. 1964, 41, 680-689.

(61). Zhang Y; Furyk S; Bergbreiter DE; Cremer PS, Specific Ion Effects on the Water Solubility of Macromolecules: PNIPAM and the Hofmeister Series. J. Am. Chem. Soc. 2005, 127, 1450514510. [PubMed: 16218647]

(62). Sun S; Wu P Infrared Spectroscopic Insight into Hydration Behavior of Poly (Nvinylcaprolactam) in Water. J. Phys. Chem. B 2011, 115, 11609-11618. [PubMed: 21899307]

(63). Cho CH; Urquidi J; Singh S; Robinson GW, Thermal Offset Viscosities of Liquid H2O, D2O, and T2O. J. Phys. Chem. B.1999, 103, 1991-1994.

(64). Kim MD; Dergunov SA; Richter AG; Durbin J; Shmakov SN; Jia Y; Kenbeilova S; Orazbekuly Y; Kengpeiil A; Lindner E Facile Directed Assembly of Hollow Polymer Nanocapsules within Spontaneously Formed Catanionic Surfactant Vesicles. Langmuir 2014, 30, 7061-7069. [PubMed: 24341533]

(65). Richter AG; Dergunov SA; Ganus B; Thomas Z; Pingali SV; Urban V; Liu Y; Porcar L; Pinkhassik E Scattering Studies of Hydrophobic Monomers in Liposomal Bilayers: An Expanding Shell Model of Monomer Distribution. Langmuir 2011, 27, 3792-3797. [PubMed: 21391646]

(66). Liu F; Kozlovskaya V; Kharlampieva E Poly(N-vinylcaprolactam): From polymer Synthesis to Smart Self-Assemblies", Ch.4 In Temperature-responsive polymers: Chemistry, Properties and Applications, Khutoryanskiy V and Georgiou T (Eds), John Wiley \& Sons, Hoboken, 2018.

(67). Higgins W; Kozlovskaya V; Alford A; Ankner J; Kharlampieva E Stratified TemperatureResponsive Multilayer Hydrogels of Poly(N-vinylpyrrolidone) and Poly(N-vinylcaprolactam): Effect of Hydrogel Architecture on Properties. Macromolecules 2016, 49, 6953-6964.

(68). Liang X; Kozlovskaya V; Chen Y; Zavgorodnya O; Kharlampieva E Thermosensitive Multilayer Hydrogels of Poly(N-vinylcaprolactam) as Nanothin Films and Shaped Capsules. Chem. Mater. 2012, 24, 3707-3719. [PubMed: 23087543]

(69). Itel F; Chami M; Najer A; Lörcher S; Wu D; Dinu IA; Meier W Molecular Organization and Dynamics in Polymersome Membranes: A Lateral Diffusion Study. Macromolecules 2014, 47, 7588-7596.

(70). Photos PJ; Bacakova L; Discher B; Bates FS; Discher DE Polymer Vesicles In Vivo: Correlations with PEG molecular Weight. J. Control. Release 2003, 90, 323-334. [PubMed: 12880699]

(71). Vorob'ev MM; Burova TV; Grinberg NV; Dubovik AS; Faleev NG; Lozinsky VI Hydration Characterization of N-vinylcaprolactam Polymers by Absorption Millimeter-wave Measurements. Colloid Polym. Sci. 2010, 288, 1457-1463.

(72). Stark F; Fallander J, AP Wright in Comprehensive Organometallic Chemistry, ed. Wilkinson G, FGA Stone and EW Abel. Pergamon, New York: 1982.

(73). Tang Y-H; Li Z; Li X; Deng M; Karniadakis GE Non-equilibrium Dynamics of Vesicles and Micelles by Self-assembly of Block Copolymers with double Thermoresponsivity. Macromolecules 2016, 49, 2895-2903.

(74). Swallow FE Viscosity of Polydimethylsiloxane Gum: Shear and Temperature Dependence from Dynamic and capillary Rheometry. J. Appl. Polym. Sci. 2002, 84, 2533-2540.

(75). Brož P; Benito SM; Saw C; Burger P; Heider H; Pfisterer M; Marsch S; Meier W; Hunziker P, Cell targeting by a generic receptor-targeted polymer nanocontainer platform. J. Control. Release. 2005, 102, 475-488. [PubMed: 15653165]

(76). Nardin C; Hirt T; Leukel J; Meier W, Polymerized ABA Triblock Copolymer Vesicles. Langmuir 2000, 16, 1035-1041. 
(a)
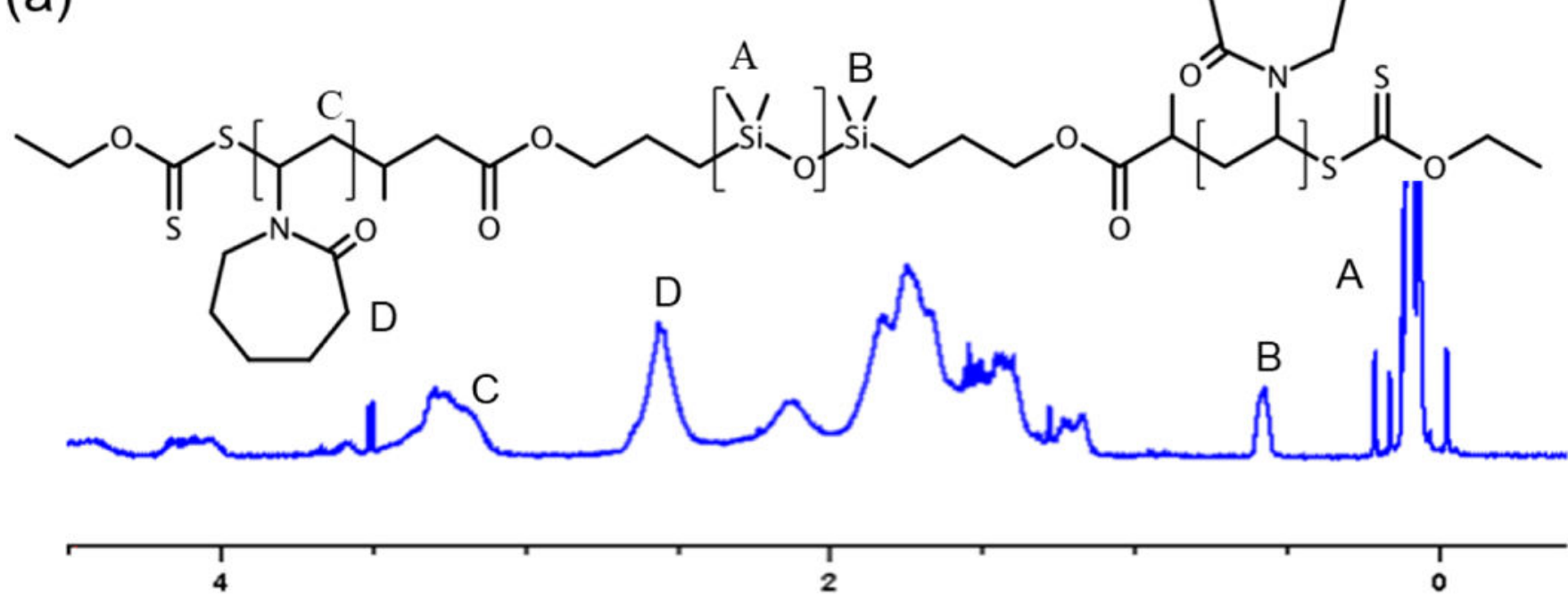

(b)
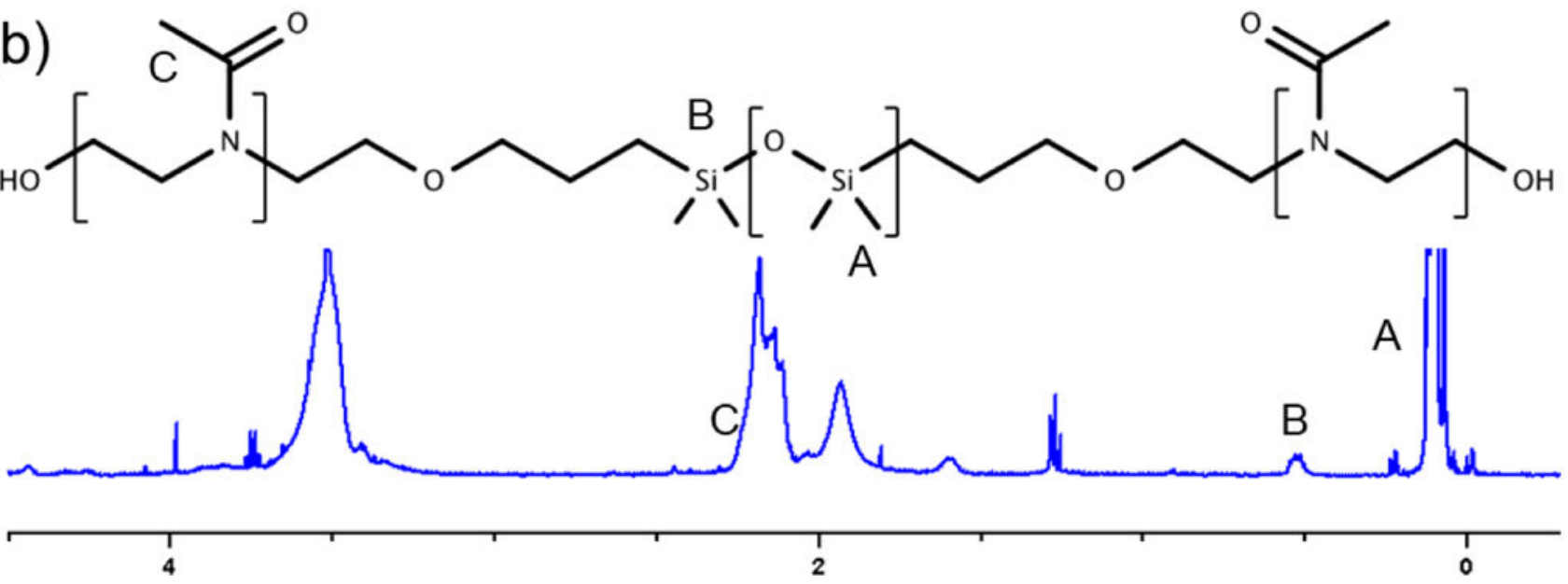

Figure 1.

Chemical structure of (a) $\mathrm{PVCL}_{5}-\mathrm{PDMS}_{30}-\mathrm{PVCL}_{5}$ and (b) $\mathrm{PMOXA}_{14}-\mathrm{PDMS}_{65}-\mathrm{PMOXA}_{14}$ triblock copolymers and their ${ }^{1} \mathrm{HNMR}$ spectra. 


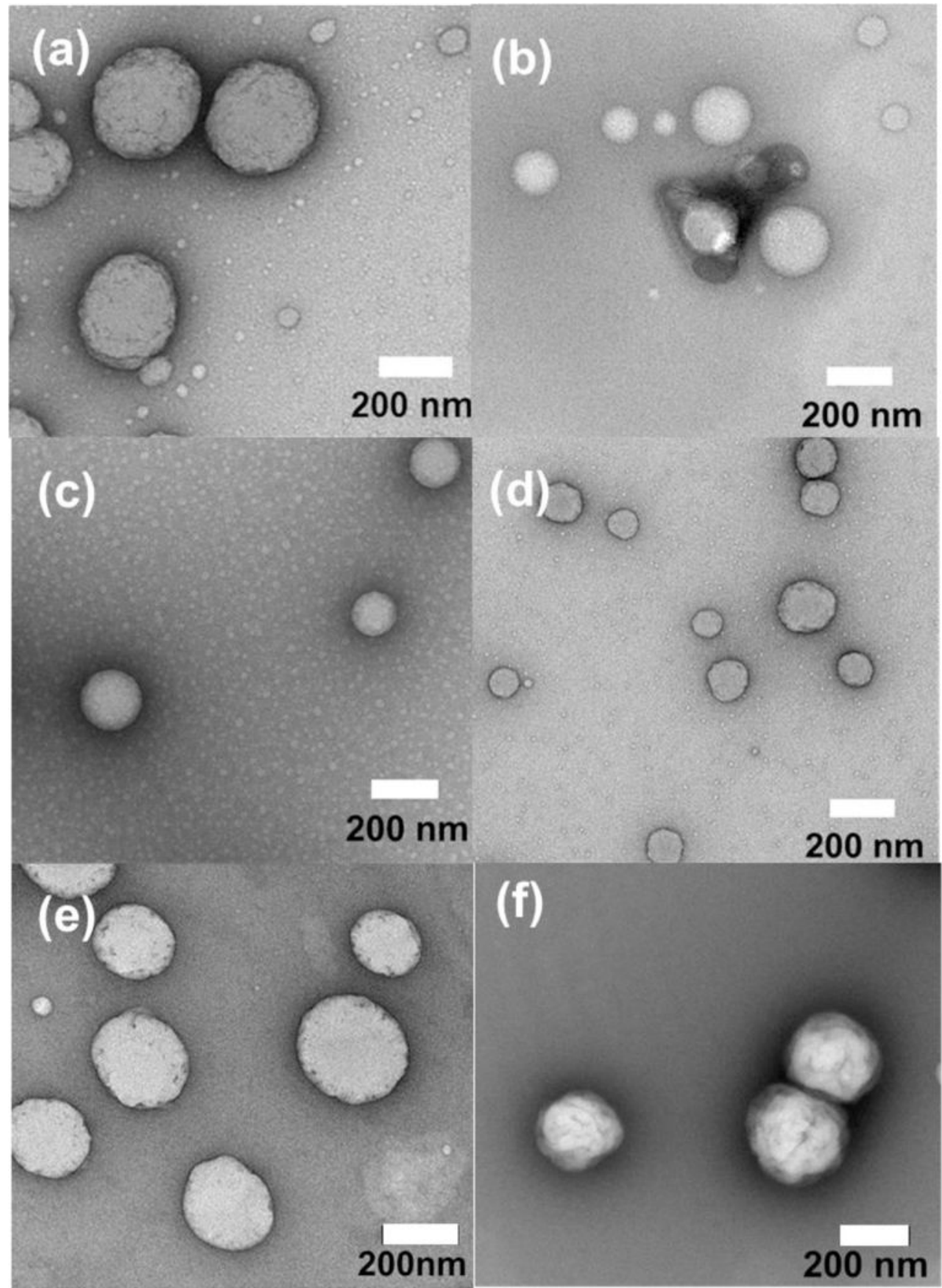

Figure 2.

TEM image of (a) $\mathrm{PVCL}_{10}-\mathrm{PDMS}_{65}-\mathrm{PVCL}_{10}$, (b) $\mathrm{PMOXA}_{14}-\mathrm{PDMS}_{65}-\mathrm{PMOXA}_{14}$, (c) $\mathrm{PVCL}_{15}-\mathrm{PDMS}_{65}-\mathrm{PVCL}_{15}$, (d) $\mathrm{PVCL}_{21}-\mathrm{PDMS}_{65}-\mathrm{PVCL}_{21}$, (e) $\mathrm{PVCL}_{7}-\mathrm{PDMS}_{47}-\mathrm{PVCL}_{7}$ and (f) $\mathrm{PVCL}_{5}-\mathrm{PDMS}_{30}-\mathrm{PVCL}_{5}$. 
a)

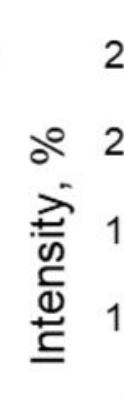

c)

e)

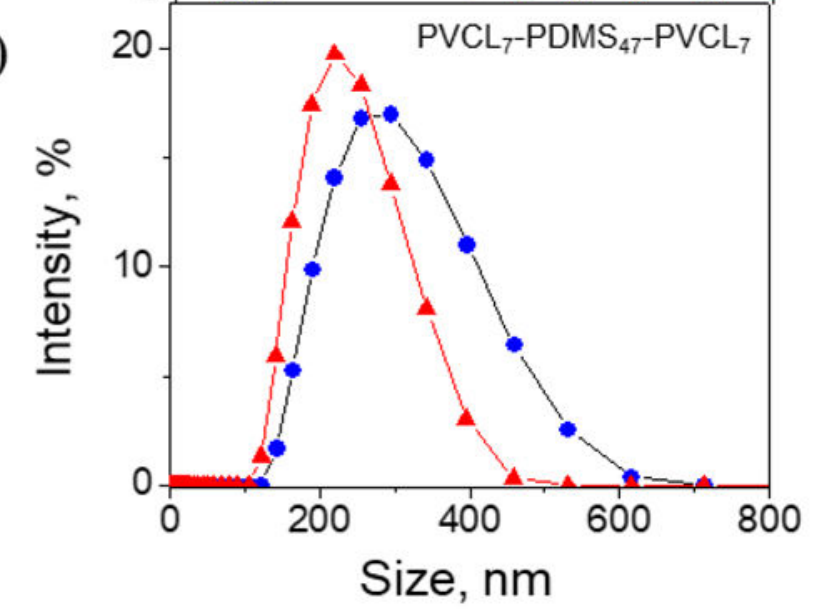

b)

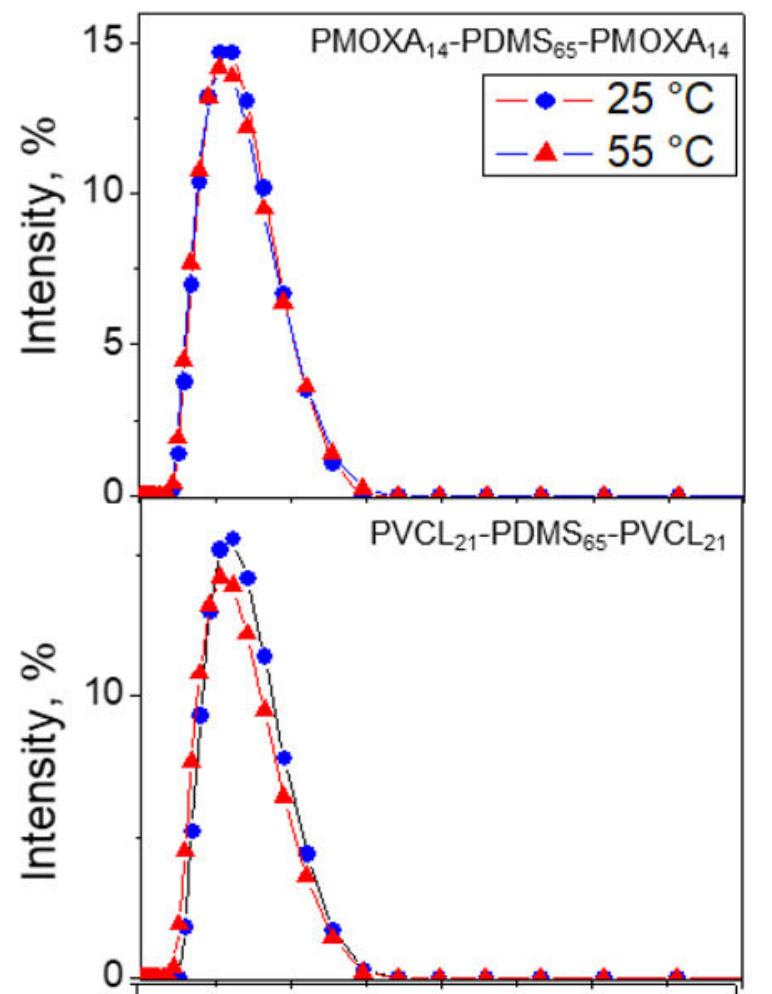

f)

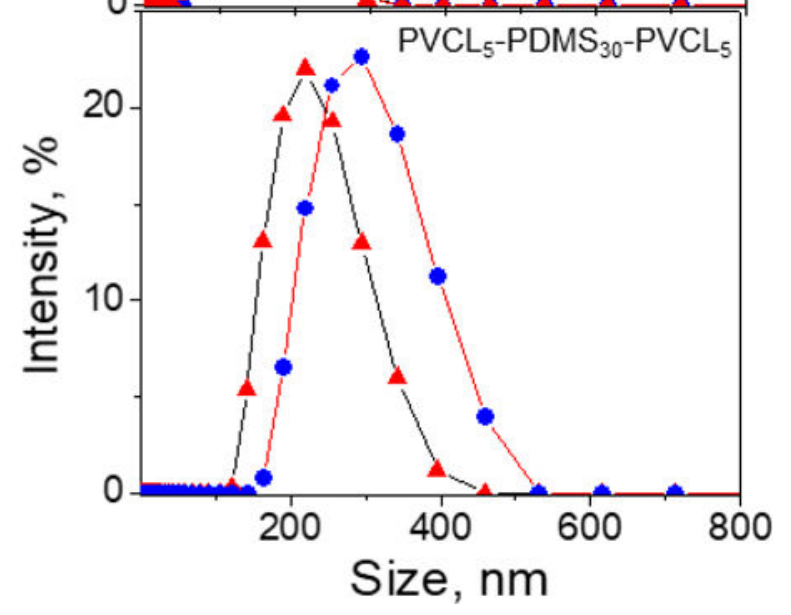

Figure 3.

Size of the vesicles from DLS at $25{ }^{\circ} \mathrm{C}(\boldsymbol{)})$ and $55^{\circ} \mathrm{C}(\boldsymbol{\Delta})$ for (a) $\mathrm{PVCL}_{10}-\mathrm{PDMS}_{65^{-}}$ $\mathrm{PVCL}_{10}$, (b) PMOXA $14-\mathrm{PDMS}_{65}-\mathrm{PMOXA}_{14}$, (c)PVCL $15-\mathrm{PDMS}_{65}-\mathrm{PVCL}_{15}$, (d)PVCL $\mathrm{PV}_{21}$ $\mathrm{PDMS}_{65}-\mathrm{PVCL}_{21}$, (e) $\mathrm{PVCL}_{7}-\mathrm{PDMS}_{47}-\mathrm{PVCL}_{7}$ and (f) $\mathrm{PVCL}_{5}-\mathrm{PDMS}_{30}-\mathrm{PVCL}_{5}$ 
a)



c)

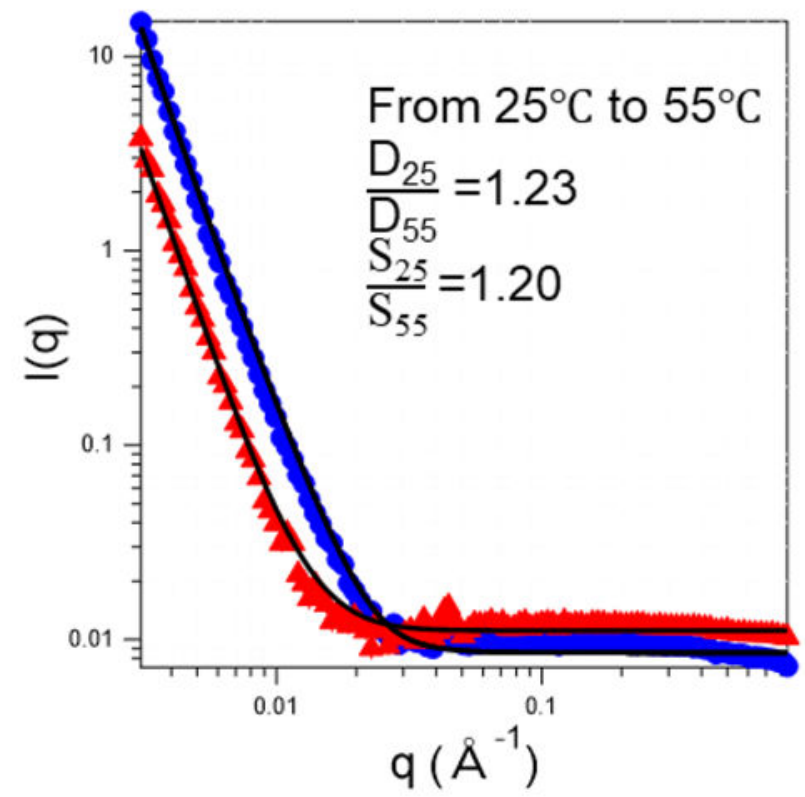

b)
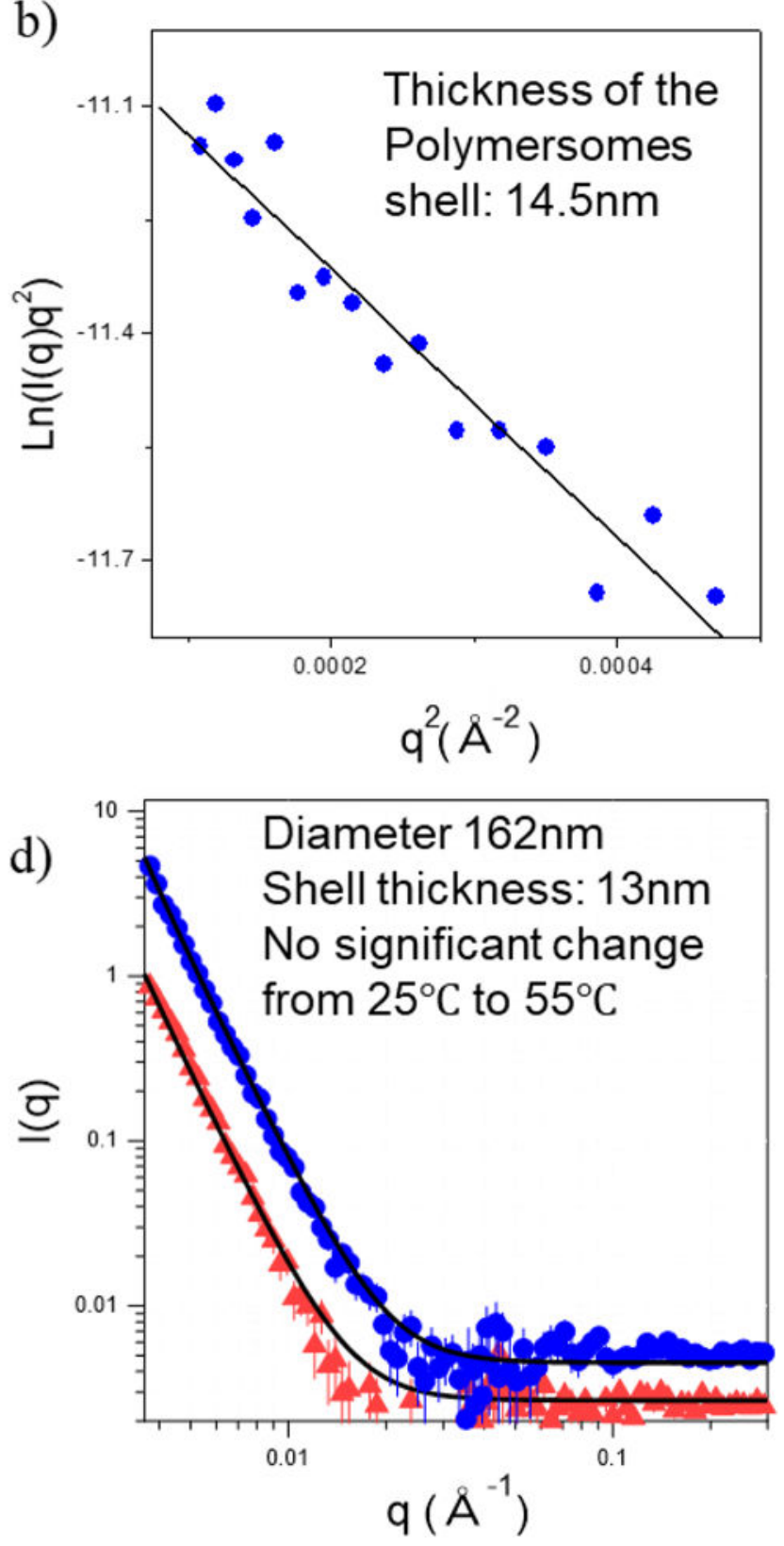

Figure 4.

(a) SANS curve of the $\mathrm{PVCL}_{10}-\mathrm{PDMS}_{65}-\mathrm{PVCL}_{10}$ vesicles $(-)$ at $25^{\circ} \mathrm{C}$ and its fit by the Guinier-Porod model (solid line) showed an Rg of $170 \mathrm{~nm}$. (b) Modified Guinier plot of the $\mathrm{PVCL}_{10}-\mathrm{PDMS}_{65}-\mathrm{PVCL}_{10}$ vesicles $(-)$ at $25^{\circ} \mathrm{C}$. The solid line is a linear fit to the data, giving a slope of $1773.4 \AA^{2}$ corresponding to a membrane thickness of $145 \AA$. SANS curve of the (c) $\mathrm{PVCL}_{10}-\mathrm{PDMS}_{65}-\mathrm{PVCL}_{10}$ vesicles at $25^{\circ} \mathrm{C}(\boldsymbol{\bullet})$ and $55^{\circ} \mathrm{C}(\boldsymbol{\Delta})$, and (d)

PMOXA $_{14}-\mathrm{PDMS}_{65}-\mathrm{PMOXA}_{14}$ vesicles at $25^{\circ} \mathrm{C}(\boldsymbol{O})$ and $55^{\circ} \mathrm{C}(\boldsymbol{\Delta})$ (vertically offset for clarity). Solid lines in both plots represent model fits by the core-shell model in (c) and (d). $\mathrm{D}_{25}$ and $\mathrm{D}_{55}$ represent the diameter at $25{ }^{\circ} \mathrm{C}$ and $55^{\circ} \mathrm{C}$, respectively. $\mathrm{S}_{25}$ and $\mathrm{S}_{55}$ represent 
the shell thickness at $25{ }^{\circ} \mathrm{C}$ and $55^{\circ} \mathrm{C}$, respectively. Diameter shrinkage was calculated by $\frac{\mathrm{D}_{25}}{\mathrm{D}_{55}}$ and shell shrinkage was calculated by $\frac{\mathrm{S}_{25}}{\mathrm{~S}_{55}}$ 

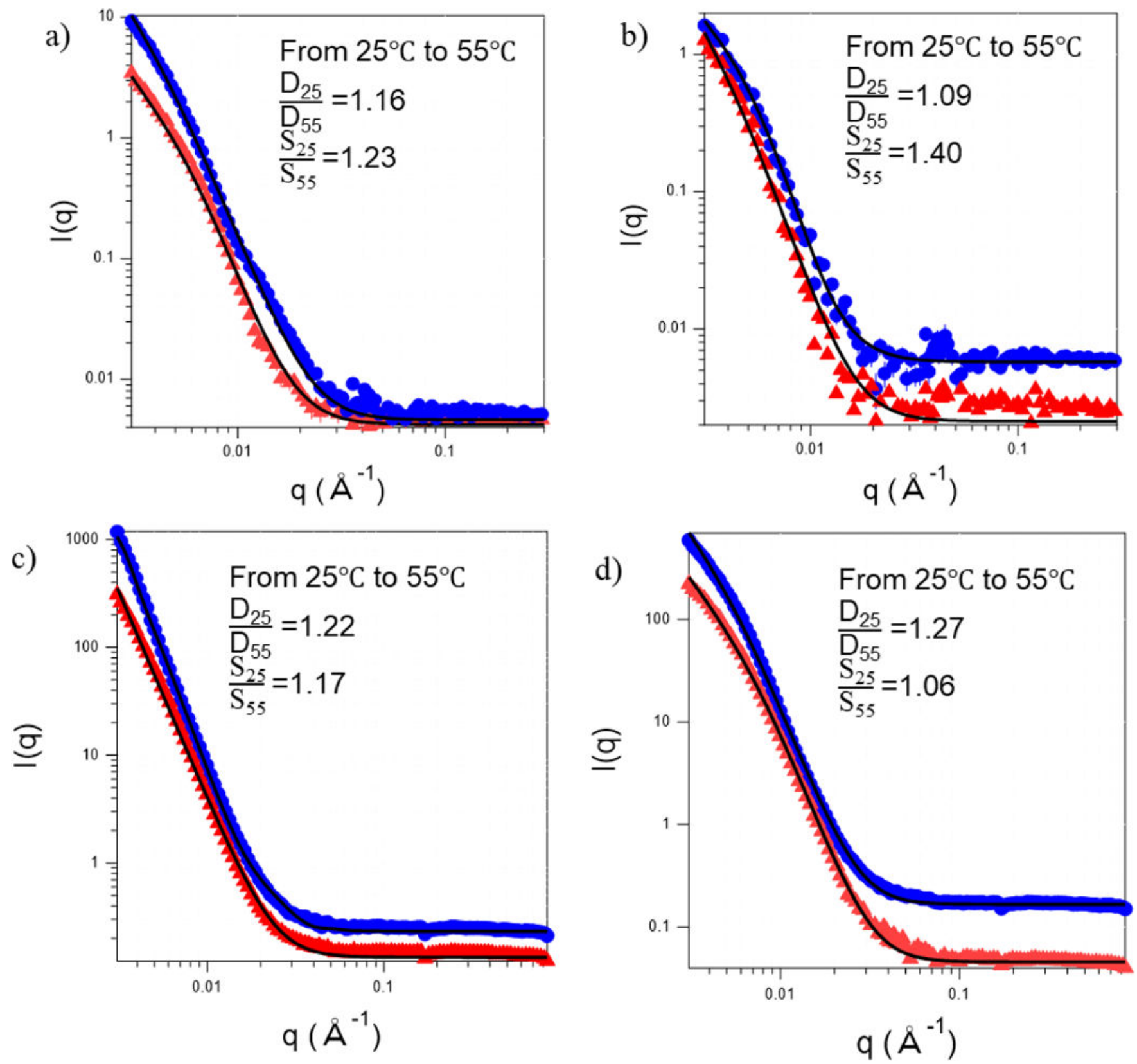

Figure 5.

SANS scattering curves of the vesicles at $25{ }^{\circ} \mathrm{C}(\boldsymbol{O})$ and $55^{\circ} \mathrm{C}(\boldsymbol{\Delta})$ for (a) $\mathrm{PVCL}_{15^{-}}$ $\mathrm{PDMS}_{65}-\mathrm{PVCL}_{15}$ and (b) PVCL $21-\mathrm{PDMS}_{65}-\mathrm{PVCL}_{21}$ for (c) $\mathrm{PVCL}_{7}-\mathrm{PDMS}_{47}-\mathrm{PVCL}_{7}$ and (d) $\mathrm{PVCL}_{5}-\mathrm{PDMS}_{30}-\mathrm{PVCL}_{5}$ Solid lines represent fitting by the core-shell model in both plots. $\mathrm{D}_{25}$ and $\mathrm{D}_{55}$ represent the diameter at $25^{\circ} \mathrm{C}$ and $55^{\circ} \mathrm{C}$, respectively. $\mathrm{S}_{25}$ and $\mathrm{S}_{55}$ represent the shell thickness at $25{ }^{\circ} \mathrm{C}$ and $55^{\circ} \mathrm{C}$, respectively. Diameter shrinkage was calculated by $\frac{D_{25}}{D_{55}}$ and shell shrinkage was calculated by $\frac{S_{25}}{S_{55}}$ 
a)

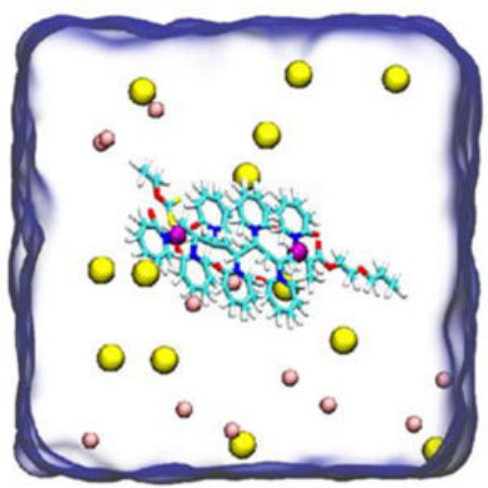

b)

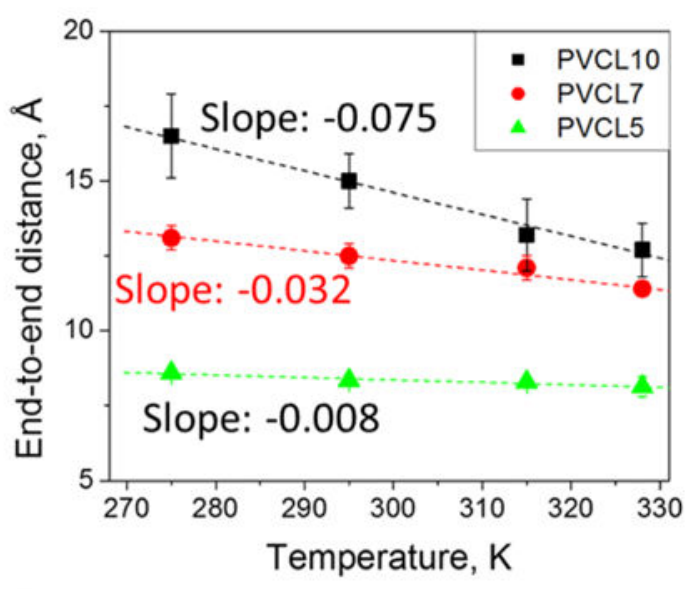

c)
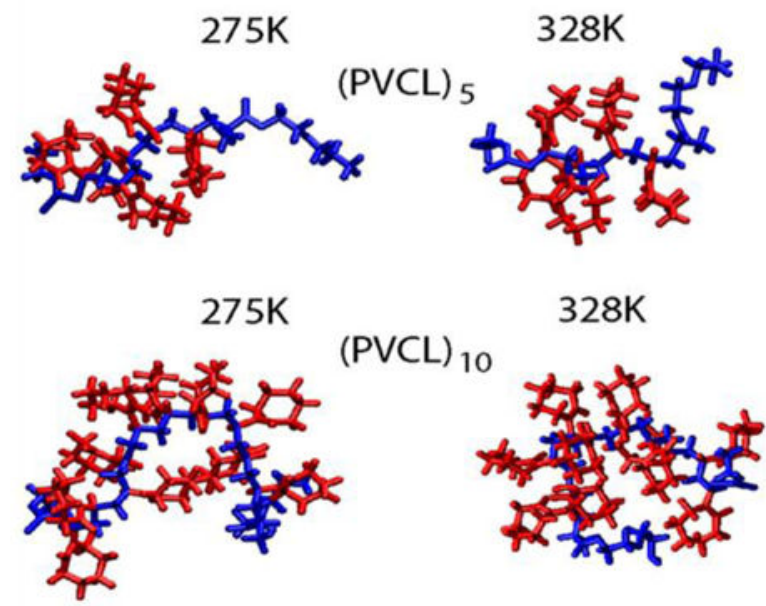

Figure 6.

MD simulations of PVCL polymers at several temperatures. (a) Typical simulation system containing one $\mathrm{PVCL}_{7}$ polymer, water and ions. $\mathrm{PVCL}_{7}$ copolymer is shown using a molecular bonds representation (carbons are shown in blue, oxygens are shown in red, hydrogens are shown in white respectively), sodium and chloride ions are shown as pink and yellow spheres respectively, water is shown as a blue semi-transparent surface. The blue spheres indicate the location of atoms used for end-to-end distance calculation. (b) Average end-to-end distance of $\mathrm{PVCL}_{5}, \mathrm{PVCL}_{7}$ and $\mathrm{PVCL}_{10}$ at 275, 295, 315 and $328 \mathrm{~K}$. Each data 
point represents an average of four independent MD simulations each lasting between 120 and $320 \mathrm{~ns}$. The error bars show the standard deviations among the average values of the four replicate simulations. Slope has units of $\mathrm{A} / \mathrm{K}$. (c) Representative conformations of $\mathrm{PVCL}_{5}$ (top row) and $\mathrm{PVCL}_{10}$ (bottom row) at $275 \mathrm{~K}$ and $328 \mathrm{~K}$. The backbone and side chains of each polymer are shown in blue and red, respectively. 
PVCL PVCL Short Chain
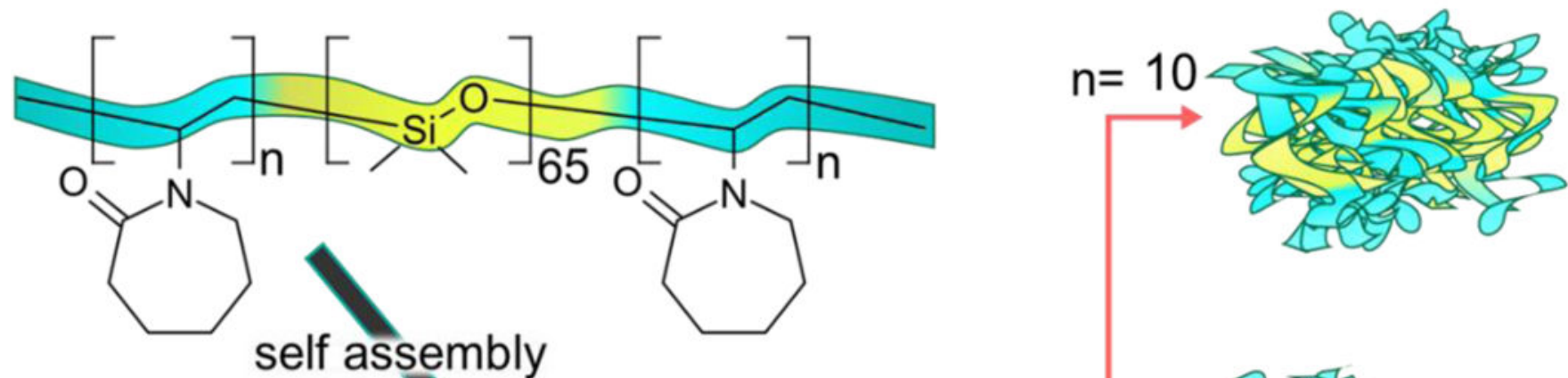

self assembly
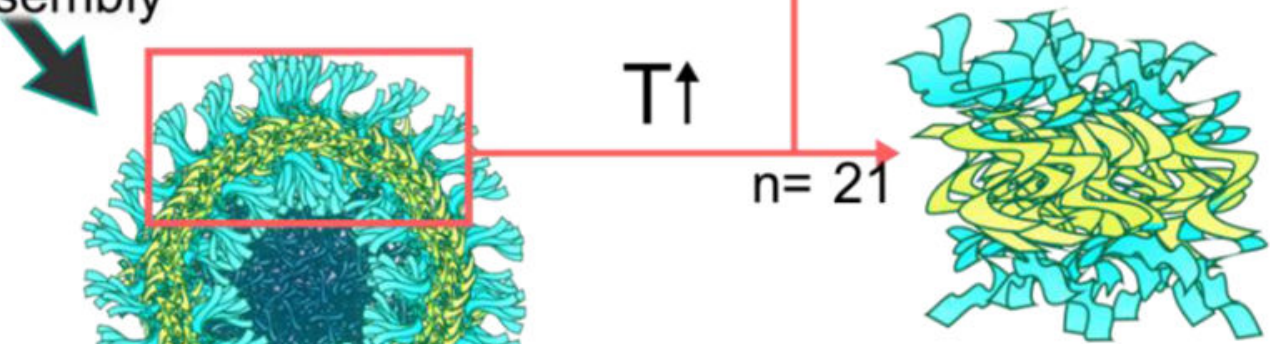

Long Chain

Figure 7.

The possible mechanism for the shell behavior at elevated temperature. Shorter PVCL chain length $(n=10)$ polymersomes exhibit interchain diffusion between PVCL and PDMS, while longer PVCL chain length $(n=21)$ display primarily interchain entanglement. 
Table 1.

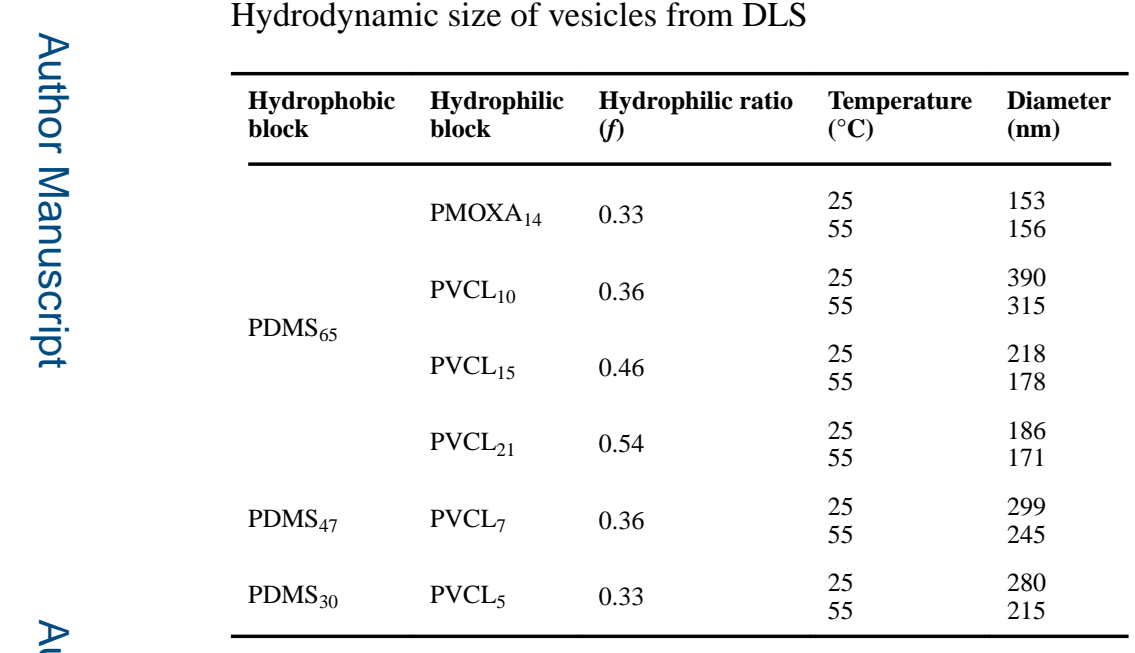


Table 2.

Vesicle parameters obtained from SANS

\begin{tabular}{|c|c|c|c|c|c|c|}
\hline & & & $25^{\circ} \mathrm{C}$ & & $55^{\circ} \mathrm{C}$ & \\
\hline $\begin{array}{l}\text { Hydrophobic } \\
\text { Block }\end{array}$ & $\begin{array}{l}\text { Hydrophilic } \\
\text { Block }\end{array}$ & $\begin{array}{l}\text { Transition } \\
\text { Temperature } \\
* \\
\left({ }^{\circ} \mathbf{C}\right)\end{array}$ & $\begin{array}{l}\text { Diameter } \\
(\mathbf{n m})\end{array}$ & $\begin{array}{l}\text { Thickness } \\
(\mathbf{n m})\end{array}$ & $\begin{array}{l}\text { Diameter } \\
(\mathbf{n m})\end{array}$ & $\begin{array}{l}\text { Thickness } \\
(\mathbf{n m})\end{array}$ \\
\hline \multirow{4}{*}{$\mathrm{PDMS}_{65}$} & $\mathrm{PMOXA}_{14}$ & none & 162 & 13 & 162 & 13 \\
\hline & $\mathrm{PVCL}_{10}$ & $40-42$ & 352 & 14.1 & 286 & 11.7 \\
\hline & $\mathrm{PVCL}_{15}$ & $35-37$ & 206 & 16 & 178 & 13 \\
\hline & $\mathrm{PVCL}_{21}$ & $35-37$ & 183 & 19.1 & 167 & 13.6 \\
\hline $\mathrm{PDMS}_{47}$ & $\mathrm{PVCL}_{7}$ & $40-42$ & 299 & 10.3 & 245 & 8.8 \\
\hline $\mathrm{PDMS}_{30}$ & $\mathrm{PVCL}_{5}$ & $40-42$ & 280 & 8.5 & 220 & 8 \\
\hline
\end{tabular}

